\title{
The Social Relations Model for Count Data
}

\section{An Exploration of Intergenerational Co-Activity Within Families}

\author{
Justine Loncke ${ }^{1}$, William L. Cook ${ }^{2}$, Jenae M. Neiderhiser ${ }^{3}$, and Tom Loeys ${ }^{1}$ \\ ${ }^{1}$ Department of Data Analysis, Ghent University, Belgium \\ ${ }^{2}$ Center for Excellence in Neuroscience, University of New England, ME, USA \\ ${ }^{3}$ Department of Psychology, The Pennsylvania State University, PA, USA
}

\begin{abstract}
The social relations model (SRM) is typically used to identify sources of variance in interpersonal dispositions in families. Traditionally, it uses dyadic measurements that are obtained from a round-robin design, where each family member rates each other family member. Those dyadic measurements are mostly considered to be continuous, but we, however, will discuss how the SRM can be adapted to count dyadic measurements. Such SRM for count data can be formulated in the SEM-framework by viewing it as a confirmatory factor analysis (CFA), but it can also be defined in the multilevel framework. These two frameworks result in equivalent models of which the parameters can be estimated using maximum likelihood estimation or a Bayesian approach. We perform a simulation study to compare the performance of those two estimators. As an illustration, we consider intergenerational co-activity data from a block design and contrast family dynamics between non-divorced families and stepfamilies.
\end{abstract}

Keywords: Bayesian analysis, family social relations model, perceived co-activity, levels of analysis, count data

Families consist of complex interpersonal relationships, that are characterized by interdependence (Kelley, 1979). To completely grasp these interpersonal processes, we must understand the reciprocal nature and context of these family relationships (Reis, Collins, \& Berscheid, 2000). This becomes possible in a holistic approach, in which families are seen as dynamic systems and where the family members form interdependent subsystems (Cox \& Paley, 2003). A model that is able to capture the family as a complex system in which family members mutually influence each other is the family social relations model (Cook, 1994; Kashy \& Kenny, 1990). It models these complex family dynamics at three different levels (i.e., individual, dyadic, and family), while taking into account the different family roles. It is a modified version of the traditional social relations model (SRM; Kenny \& La Voie, 1984) and typically makes use of continuous dyadic measurements that are obtained from a round-robin design. It has also been used to analyze ratings from a block design as well as ratings from specific family subsystems, for example, cohesiveness in mother-child, father-child, and fathermother dyads (Cook \& Kenny, 2006).

The main challenge that we will tackle in this paper lies in the nature of the dyadic measurements obtained from a block or round robin design. Up until now, most SRM research is limited to the decomposition of continuous dyadic measurements. In the present study, we accommodate the family SRM to count outcomes as well. Typically, the parameters of the family SRM are estimated using modeling strategies that by default rely on the use of the maximum likelihood (ML) framework, that is, structural equation modeling (SEM) (Kenny, Kashy, \& Cook, 2006; Stas, Schönbrodt, \& Loeys, 2015) and multilevel modeling (Rasbash, Jenkins, O'Connor, Tackett, \& Reiss, 2011; Snijders \& Kenny, 1999). The SRM parameters, however, can also be estimated in a Bayesian framework (Gill \& Swartz, 2007). Currently the Bayesian estimator can only be derived using the multilevel framework. Such Bayesian approach has already been proposed for the SRM without family roles, where the continuous outcomes are obtained according to a round robin design (Lüdtke, Robitzsch, Kenny, \& Trautwein, 2012) and for categorical dyadic measurements (Hoff, 2015), such as count measurements (Koster \& Leckie, 2014; Koster, Leckie, Miller, \& Hames, 2015). Instead of focussing on the SRM without roles, we will explore the SRM with family roles and accommodate it to count outcome variables. Although previous research indicated that the Bayesian approach seems promising in the 
estimation of the SRM without roles, it also showed that for continuous outcomes the Bayesian approach can result in biased estimators of the variances for small cluster sizes in combination with small sample sizes (Lüdtke et al., 2012). Since the family SRM always entails a small group size, one may wonder whether the performance of the Bayesian approach is problematic, especially when considering count data? And if so, is the ML-estimator a more adequate alternative? To answer these questions, we perform a simulation study where the performance of the Bayesian approach is compared to the performance of the ML-estimator.

As an illustrating example throughout this paper, we consider data on intergenerational co-activity within families. We use co-activity data from non-divorced families and stepfamilies that participated in the NEAD study (Nonshared Environment in Adolescent Development; Neiderhiser, Reiss, \& Hetherington, 2007). In this study each parent was asked separately to each of the two children about certain types of activities, such as going for a walk with the child in the past month. The same question was asked of each of the two children, regarding activities with each parent. A measure of intergenerational co-activity is obtained by counting the number of different activities that a family member reported with another family member. As such, the outcome reflects the perception of the rater on the number of shared activities within the past month. Using the SRM, we can then identify the levels (family, individual, and dyadic) that explain most variability in perceived co-activity between families, and investigate whether the family dynamics of perceived co-activity are different between non-divorced families and stepfamilies.

This article is organized as follows. We start with an introduction to the SRM in a block design. We first show how the SRM can be modeled as a confirmatory factor analysis (CFA) in the SEM-framework or by formulating it in the multilevel framework. Next, we show how the parameter estimates can be obtained by using ML or a Bayesian approach. Then a detailed description of the NEAD study is given. Subsequently, we use the co-activity

(A)

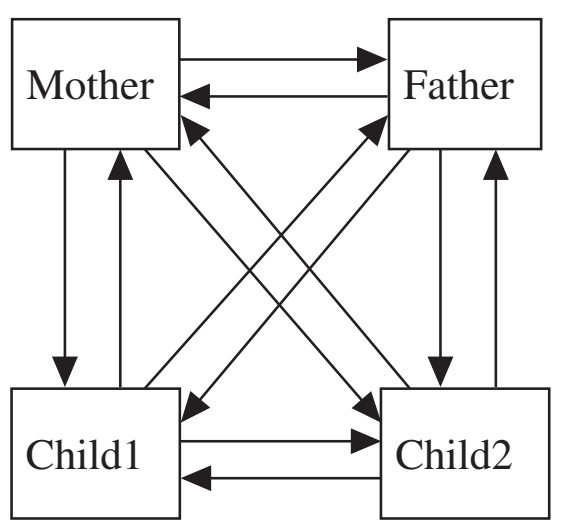

data as an inspiration source for our simulation study where the ML-estimators are compared to the Bayesian estimators of the SRM-effects. Accordingly, the approaches are illustrated using the co-activity data. The analyses described throughout the article can easily be replicated by the reader using the code found in Electronic Supplementary Material (ESM 1). We end with a discussion of our findings.

\section{The Blocked Family Social Relations Model}

The SRM traditionally analyzes data gathered according to a round robin design. Such design typically involves three or four family members (Kenny et al., 2006). When members of a four-person family are asked to rate each other's behavior 12 dyadic measurements or scores are obtained (Figure 1A). The SRM decomposes these measurements into a family effect, a perceiver and target effect (both at the individual level), and a relation-specific effect (at the dyadic level). The family effect reflects the average dyadic measurement. The perceiver effect reflects how a family member tends to perceive the others; this effect is sometimes referred to as the actor effect as well. The target or partner effect reflects how a particular family member is generally seen by the other members. Lastly, the relationship effect captures how a perceiver uniquely sees the target, while controlling for family, perceiver, and target effects.

However, sometimes family researchers are interested in family dynamics that are based on only a particular subset of relationships, for example, parent-adolescent interactions. To increase the efficiency of data collection, the dyadic measurements can then be obtained from a block design. In a block design, subjects are divided into subgroups and members of each subgroup rate all the members of the other subgroup (Kenny et al., 2006). The most natural subgroups in a family setting are the different generations, with parents rating their children and vice

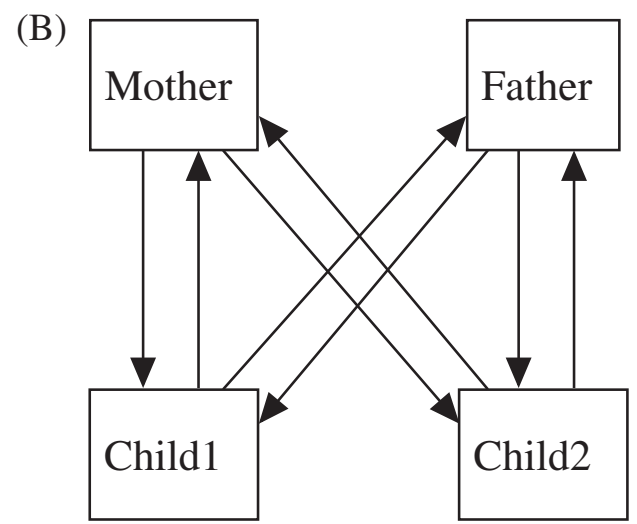

Figure 1. Designs applied in the SRM. (A) Round robin design; (B) Block design. 


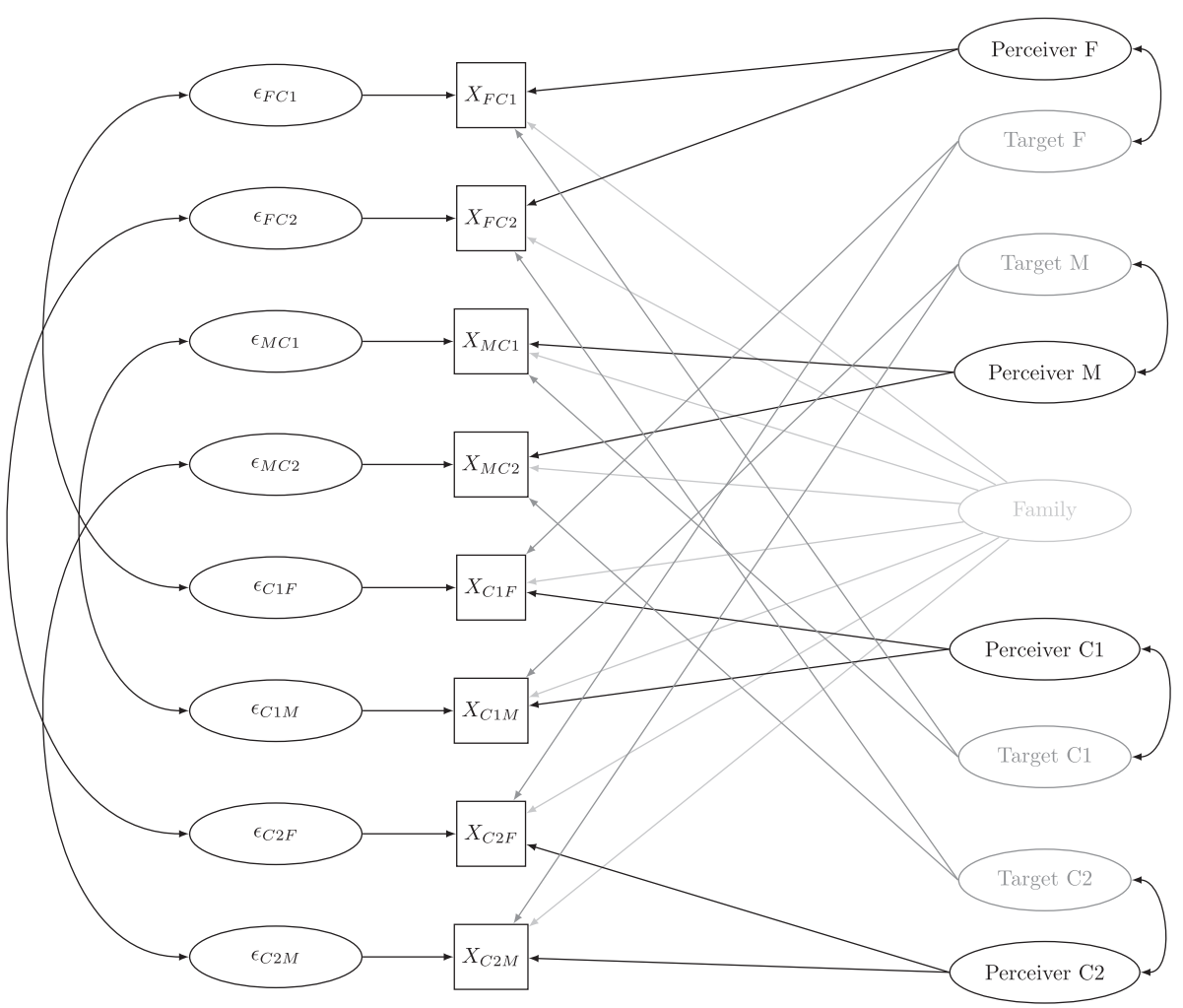

Figure 2. CFA-model for the block social relations model. versa (Figure 1B). When members of a four-person family are asked to rate each other's behavior according to such a block design eight intergenerational dyadic measurements are obtained. If the SRM is applied to analyze data from such a design, it also allows to make a distinction between the roles of the different members within the subgroups (Kenny et al., 2006). Meaning that it allows, for example, for a distinction between the dyadic measurements of the father and the mother.

Typically, estimation of the parameters of the family SRM relies on structural equation modeling (SEM) by approaching the SRM-analysis as a CFA (Kenny et al., 2006; Stas et al., 2015). Alternatively, the SRM may be fitted as a multilevel model (Rasbash et al., 2011; Snijders \& Kenny, 1999). In the following, we will show how the SRM for dyadic measurements from a block design can be modeled as a CFA in the SEM-framework or by formulating it in the multilevel framework. Note that the dyadic measurements from a round robin design are modeled in a similar fashion.

\section{SEM-Framework}

The SRM decomposes the (intergenerational) dyadic measurements $\left(X_{i j}\right)$ into four different latent effects: a family effect, a perceiver effect, a target effect, and a relation-specific effect. In a four-person family the indices $i$ and $j$ represent the father $(\mathrm{F})$, mother (M), child 1 (C1) or child 2 (C2). In our illustration $X_{\mathrm{MC} 1}$, for example, is the number of different activities that the mother reported with child 1 . Self-ratings (i.e., $i=j$ ) and intragenerational ratings are not considered here.

Figure 2 illustrates this decomposition within the SEMframework, where the SRM effects are specified as latent variables. The arrows point from the latent variables toward the dyadic measurements, illustrating how the latter are influenced by the former. The dyadic measurement $X_{\mathrm{MC} 1}$, for example, is a formation of the family effect, the mother's perceiver effect, the target effect of child 1 and the motherchild 1 relationship effect. In the CFA-model, this dyadic measurement is thus allowed to load on the family effect, the perceiver effect of the mother, the target effect of child 1 and the mother-child 1 relationship-effect. Typically, factor loadings between the dyadic measurements and the latent variables are all fixed to one in the SRM analysis.

Note, that with only one indicator per dyadic measurement the relationship effect cannot be disentangled from the measurement error. To disentangle the relationship effect from the measurement error at least two indicators of each dyadic relation are needed (Back \& Kenny, 2010). However, the use of two indicators is sometimes avoided in SRM-applications with small samples, because it can lead to unstable estimates of the components (Stas et al., 2015). In this article, we will consider only one observation per dyadic measurement. This implies that the relationship effects will be systematically overestimated and thus cannot be interpreted unambiguously. 
In general, SRM components are assumed to be independent, but some are related to each other through patterns of reciprocity (Kenny et al., 2006). Generalized reciprocity reflects the correlation between a person's perceiver and target effect (e.g., capturing whether the number of activities perceived by the mother is associated with the number of activities that the children perceived in relation to the mother). Dyadic reciprocity reflects the correlation between the relationship effects that represent two sides of a certain relationship (e.g., capturing whether the number of $\mathrm{M}-\mathrm{C} 1$ relation-specific activities is associated with the number of C1-M relation-specific activities). These two types of reciprocity are indicated in Figure 2 by two-headed arrows. For data such as perceived co-activity measures, reciprocity correlations are more accurately conceptualized as agreement in the perspectives of the respondents. We will, however, use the term reciprocity to be consistent with the SRM literature.

Finally, we discuss the mean-structure of the SRM. In the SRM for dyadic measurements from a block design, we have a total of 17 SRM means (one family mean, four perceiver means, four target means, and eight relationship means) while only eight means are observed. Therefore to identify the model, restrictions are needed. The family mean can be defined as the average over the eight dyadic measurements. Furthermore mean perceiver effects and mean target effects are assumed to sum to zero. By design, the sum of the parents' mean perceiver effects equals the sum of the children's mean target effects. Restrictions are further applied on the relationship effects such that the mean SRM relationship effects sum to zero for a given perceiver or a given target. With those restrictions, the means of the SRM-effects are just identified.

\section{Multilevel Framework}

Alternatively, the SRM can be fitted in the multilevel framework (Rasbash et al., 2011; Snijders \& Kenny, 1999). Note that the SRM in the multilevel framework is equivalent to the SRM in the SEM framework (Rovine \& Molenaar, 2000). Here, the dyadic measurement $\left(X_{i j}\right)$ is modeled as a combination of the random SRM effects:

$$
X_{i j}=\mu_{i j}+b_{\mathrm{Fam}}+b_{\mathrm{Per}_{i}}+b_{\operatorname{Tar}_{j}}+\epsilon_{i j},
$$

where $\mu_{i j}$ denotes the expected number of perceived co-activities by role $i$ with role $j, b_{\text {Fam }}$ denotes the family effect, $b_{\text {Per }_{i}}$ denotes the perceiver effect of role $i, b_{\text {Tar }_{j}}$ denotes the target effect of role $j$, and $\epsilon_{i j}$ denotes the measurement error. Note that no relationship effects are explicitly incorporated in the model, since those can only be separated from error measurement when there are multiple dyadic measurements available.
In the multilevel perspective, the SRM effects are random effects that are assumed to be (bivariate) normally distributed:

$$
\begin{gathered}
b_{\mathrm{Fam}} \sim N\left(\mathrm{0}, \sigma_{\mathrm{Fam}^{2}}^{2}\right), \\
\left(\begin{array}{l}
b_{\operatorname{Per}_{i}} \\
b_{\operatorname{Tar}_{\mathrm{i}}}
\end{array}\right) \sim N\left(\left[\begin{array}{l}
0 \\
0
\end{array}\right], \quad\left[\begin{array}{cc}
\sigma_{\operatorname{Per}_{i}}^{2} & \rho_{i} \sigma_{\operatorname{Per}_{i}} \sigma_{\operatorname{Tar}_{i}} \\
\rho_{i} \sigma_{\operatorname{Per}_{i}} \sigma_{\operatorname{Tar}_{i}} & \sigma_{\operatorname{Tar}_{i}}^{2}
\end{array}\right]\right),
\end{gathered}
$$

where $\sigma_{\mathrm{Fam}}^{2}$ measures the family variance, $\sigma_{\mathrm{Per}_{i}}^{2}$ measures the perceiver variance of role $i, \sigma_{\operatorname{Tar}_{i}}^{2}$ measures the target variance of role $i$, and $\rho_{i} \sigma_{\operatorname{Per}_{i}} \sigma_{\operatorname{Tar}_{i}}$ measures the generalized reciprocity for role $i$. This formulation clearly shows that we have role-specific distributions for the perceiver and target effects.

Under the scenario of dyadic measurements at the interval level, the residuals are assumed to be bivariate normally distributed:

$$
\left(\begin{array}{c}
\epsilon_{i j} \\
\epsilon_{j i}
\end{array}\right) \sim N\left(\left[\begin{array}{l}
0 \\
0
\end{array}\right], \quad\left[\begin{array}{cc}
\sigma_{\epsilon_{i j}}^{2} & \rho_{i j} \sigma_{\epsilon_{i j}} \sigma_{\epsilon_{j i}} \\
\rho_{i j} \sigma_{\epsilon_{i j}} \sigma_{\epsilon_{j i}} & \sigma_{\epsilon_{j i}}^{2}
\end{array}\right]\right),
$$

where $\sigma_{\epsilon_{i j}}^{2}$ measures the relationship variance between perceiver $i$ and target $j$, and $\rho_{i j} \sigma_{\epsilon_{i j}} \sigma_{\epsilon_{i j}}$ measures the dyadic reciprocity.

Under the scenario of count dyadic measurements, a Poisson distribution for $X_{i j}$ given the random SRM effects can be assumed, with mean $\mu_{i j}$. Ideally a bivariate Poisson distribution should be assumed for $X_{i j}$ and $X_{j i}$, but practical implementation is lacking in most software. Hence, we will leave the dyadic reciprocity unspecified. Note that for ease of exposition we focus on the Poisson distribution, but one should be aware of its strong assumptions (Loeys, Moerkerke, De Smet, \& Buysse, 2012). It assumes the mean and the variance to be equal and does not allow for frequent zero-valued observations (i.e., zero-inflation). Negative binomial distributions or zero-inflated count models could be used as alternatives to overcome these issues.

As mentioned before, SRM-researchers are mainly interested in the relative importance of the SRM effects as sources of variation in the dyadic measurements. To this end, they calculate the variance partition coefficient (VPC) of each SRM effect for each dyadic measurement. The VPCs for normally distributed dyadic measurements can easily be obtained by dividing each estimated variance by the total of the four estimated variances. For example, the amount of variability in $X_{\mathrm{MC} 1}$ attributed to the variance in the perceiver effect of the mother, is than given by

$$
\mathrm{VPC}_{\text {Per }_{\mathrm{M}}}=\frac{\sigma_{\text {Per }_{\mathrm{M}}}^{2}}{\sigma_{\mathrm{Fam}}^{2}+\sigma_{\text {Per }_{\mathrm{M}}}^{2}+\sigma_{\text {Tar }_{\mathrm{C} 1}}^{2}+\sigma_{\epsilon_{\mathrm{MC}}}^{2}} .
$$


The VPCs of count dyadic measurements are more complex. Austin, Stryhn, Leckie, and Merlo (2018) recently proposed a VPC for count outcomes. Using their expression, the variability in $X_{\mathrm{MC} 1}$ attributed to either the family effect, the perceiver effect of the mother or the target effect of child 1 is than given by equation (6) at the bottom of the page.

Using a first order Taylor expansion for $\exp \left(\sigma_{\mathrm{Fam}}^{2}+\right.$ $\sigma_{\text {Per }_{\mathrm{M}}}^{2}+\sigma_{\mathrm{Tar}_{\mathrm{C}}}^{2}$ ), this expression to the following equation (7) below.

This allows us to obtain a first order approximation of the variance that can be attributed to the perceiver effect of the mother alone, as decribed in equation (8) at the bottom of the page.

Similar as in the SEM-framework, the eight means $\mu_{i j}$ can be specified in terms of a family mean $\left(\beta_{\text {Fam }}\right)$, three perceiver means $\left(\beta_{\mathrm{Per}_{i}}\right)$, three target means $\left(\beta_{\mathrm{Tar}_{i}}\right)$ and a relation-specific mean $\left(\beta_{\mathrm{Re}_{i j}}\right)$.

\section{Estimators for High Dimensional Random Effects}

The parameters of the SRM model for dyadic scores at the interval level can easily be estimated using ML estimation. ML estimation is used by default by popular SEM packages, such as Mplus (Muthén \& Muthén, 2012) or lavaan (Rosseel, 2012), and by multilevel packages, such as lme4 (Bates, Mäechler, Bolker, \& Walker, 2015) or nlme in R (Pinheiro, Bates, DebRoy, Sarkar, \& R Core Team, 2018). Despite the equivalence between the SEM and multilevel framework, SEM software is currently a better choice for estimating the SRM model; because multilevel software does not easily allow to define constraints on the meanstructure. Although the ML-estimator is used by default by many researchers, it does have some general limitations:
(1) in a small sample size there is no guarantee that the point estimates are unbiased (Lee \& Song, 2004) and (2) improper estimates (i.e., Heywood cases) might be obtained for a complex SEM-model such as the SRM (Nevitt \& Hancock, 2004). The ML-estimator, moreover, has an additional limitation in the non-normal case: the computation time increases exponentially with increasing number of latent effects, because integrating out the latent effects becomes analytically intractable when dealing with nonnormal outcomes. In that case, the likelihood function is tackled by either approximating the integrand or the integral itself (Tuerlinckx, Rijmen, Verbeke, \& De Boeck, 2006). When considering a Poisson distribution the integration becomes analytically intractable, and one may have to rely on Monte Carlo integration to evaluate the integrand (Tuerlinckx et al., 2006). This method is particularly useful for higher-dimensional integrals and is easily available in the SEM software Mplus.

Alternatively, the parameters of the SRM model can be estimated using a Bayesian approach. Such Bayesian approach has the potential to overcome some of the shortcomings of the ML-estimator. First, sampling-based Bayesian approaches allow one to make reliable inferences about variance components even in small sample sizes. Second, improper estimates may occur less in the Bayesian approach, if the parameter space of the model is correctly specified. Lastly, with an increasing number of latent variables, the Bayesian approach is computationally faster than the ML-estimator. Especially, Gibbs sampling is well suited for the estimation of such high dimensional model (Lüdtke et al., 2012). SEM-packages such as blavaan (Merkle \& Rosseel, 2018) and Mplus have a Bayesian estimator using Gibbs sampling available. However, both blavaan and the Bayesian Mplus can currently not deal with count variables. By defining the SRM as a multilevel model, Bayesian software such as JAGS (Plummer, 2009) can easily perform

$$
\begin{aligned}
& \mathrm{VPC}_{\mathrm{MC} 1}=
\end{aligned}
$$

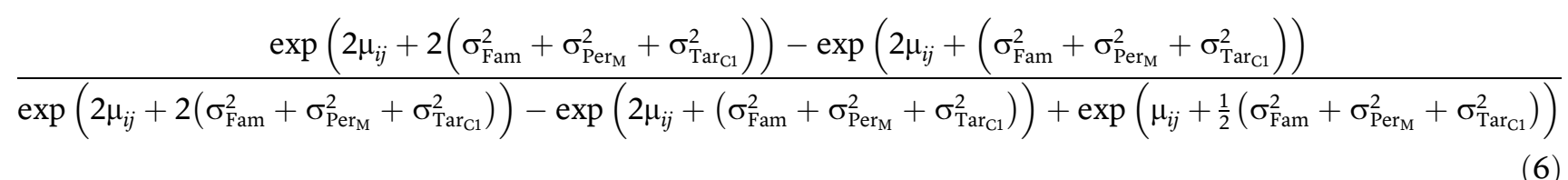

$$
\begin{aligned}
& \mathrm{VPC}_{\mathrm{MC} 1}=\frac{\exp \left(2 \mu_{i j}\right)\left(\sigma_{\mathrm{Fam}}^{2}+\sigma_{\text {Per }_{\mathrm{M}}}^{2}+\sigma_{\mathrm{Tar}_{\mathrm{C} 1}}^{2}\right)}{\exp \left(2 \mu_{\mathrm{ij}}\right)\left(\sigma_{\mathrm{Fam}}^{2}+\sigma_{\text {Per }_{\mathrm{M}}}^{2}+\sigma_{\mathrm{Tar}_{\mathrm{C} 1}}^{2}\right)+\exp \left(\mu_{i j}\right)\left(1+\frac{1}{2}\left(\sigma_{\mathrm{Fam}}^{2}+\sigma_{\text {PerM }_{\mathrm{M}}}^{2}+\sigma_{\text {Tar }_{\mathrm{C} 1}}^{2}\right)\right)} . \\
& \mathrm{VPC}_{\text {PerM }_{\mathrm{M}}}=\frac{\exp \left(2 \mu_{i j}\right)\left(\sigma_{\text {PerM }_{\mathrm{M}}}^{2}\right)}{\exp \left(2 \mu_{i j}\right)\left(\sigma_{\mathrm{Fam}}^{2}+\sigma_{\text {PerM }_{\mathrm{M}}}^{2}+\sigma_{\mathrm{Tar}_{\mathrm{C} 1}}^{2}\right)+\exp \left(\mu_{i j}\right)\left(1+\frac{1}{2}\left(\sigma_{\mathrm{Fam}_{1}}^{2}+\sigma_{\text {Per }_{\mathrm{M}}}^{2}+\sigma_{\text {Tar }_{\mathrm{C} 1}}^{2}\right)\right)} .
\end{aligned}
$$


Gibbs sampling. Note that blavaan also makes use of JAGS to perform Gibbs sampling. However, the interface of blavaan allows the user to define the model as a SEM, while the way the model is defined within JAGS itself leans more naturally towards the multilevel framework. As mentioned before both modeling strategies should result in identical models, the only difference lies in the way the model is being explicitly defined. For a detailed description on the Bayesian sampling process for the SRM (without roles) in the multilevel framework, we refer to Lüdtke et al. (2012).

In JAGS the user only needs to specify the model, which includes the decision of the likelihood and prior distributions. In the case of the SRM, the eight likelihood distributions of the dyadic outcomes are specified in accordance with their model-implied distribution. These distributions all have means that embody the expected value of the dyadic measurement as a combination of the SRM effects. To obtain a Bayesian solution, data augmentation takes place in Gibbs sampling by treating the individual random effects (i.e., the SRM effects) as hypothetical observed variables that are normally distributed and also have likelihood distributions with a certain mean and (co)variance. Their posterior means and (co)variances are the parameters of interest. Usually, non-informative prior distributions are specified for these parameters. For the priors of the means it is important to take into account the restrictions that are needed on the SRM mean-structure. Typically, in Gibbs sampling a conjugate prior, such as the Wishart prior, is used for the precision matrices. However, some caution is needed, since the Wishart prior is in fact not completely a non-informative prior. Specifically, a Wishart prior with a scale matrix that resembles the true underlying (co)variance matrix will result in less biased variance estimates (Kass \& Natarajan, 2006). In practice the underlying (co)variance matrix is not known and we will need to conduct a sensitivity analysis on the specification of the prior to evaluate the effect of different scale matrices on the (co)variance estimates (Schuurman, Grasman, \& Hamaker, 2016).

\section{NEAD Study}

\section{Sample}

The data were collected as part of the NEAD study in the United States. The original sample size consists of 720 four-member families (i.e., mother, father, an older sibling, and a younger sibling, further abbreviated as "M," "F," "C1," and "C2," respectively), who were recruited through random digit dialing and market panels (Neiderhiser et al., 2007). The dataset included five sibling types that are residing in two types of families (i.e., non-divorced families and stepfamilies): monozygotic twins, dizygotic twins, and non-twin full siblings in non-divorced families and non-twin full, half and genetically unrelated siblings in stepfamilies. Since non-twin full siblings is the only sibling type that is present in both types of families, we will consider in the current study only the 276 families that consist of non-twin full siblings (Hetherington et al., 1999). There were 94 nondivorced families and 182 stepfamilies. In the stepfamilies the biological parent was always the mother. By design the siblings are of the same sex and there were 133 brotherand 143 sister-pairs. The children were between 10 and 18 years with siblings 4 years or less apart in age. The younger siblings were, on average, 13 years $(S D=1.96)$ and the older siblings were, on average, 15 years $(S D=1.94)$.

\section{Measure}

Measures of perceived intergenerational co-activity were obtained using the expression of affection questionnaire (EAF; Hetherington \& Clingempeel, 1992). This questionnaire is often used to obtain an indication for affective family processes, such as warmth and support (Neiderhiser et al., 2007). The EAF is applied according to a block design, meaning that members of each generation rate the coactivity with members of the other generation. Since there are only four-person families included in the current study, this resulted in eight dyadic ratings of co-activity per item per family. Among other, the EAF questionnaire contains 10 items on specific activities that occur between parents and children (e.g., "Have you played a musical instrument, sang together or listened to music together in the past month with your mother/father?"). The family members were asked to indicate whether this activity had occurred at all during the last month $(0=$ no, $1=$ yes $)$. The sum of those 10 items reflects the diversity of co-activity over the past month with other family members as perceived by the rater.

\section{Simulation Study}

\section{Technical Details}

In the simulation study, we evaluate which estimator performs best in estimating the SRM means and (co-)variances for count data. We compare the performance of the ML-estimator with the performance of Bayesian estimator. Our simulation study is designed to generate Poisson distributed count data that resemble the co-activity data from the case study. The true population parameters approximately mimic the NEAD-data. In the data-generating model the variance of the family effect on the log scale $\sigma_{\text {Fam }}^{2}$ is set to 0.05 , the variances of all the perceiver effects on the log scale $\sigma_{\mathrm{Per}_{i}}^{2}$ are set to 0.5 and the variances of all 
the target variances on the log scale $\sigma_{\mathrm{Tar}_{i}}^{2}$ are set to 0.05 . Further, the $\log$ of the mean of the family effect $\beta_{\text {Fam }}$ is set to 1 and the log of the means of the perceiver and target effects, that is, $\beta_{\operatorname{Per}_{i}}$ and $\beta_{\operatorname{Tar}_{i}}$, are all set to 0.1 or -0.1 so that the above-mentioned restrictions are satisfied. Additionally the means of the relationship effects $\beta_{\operatorname{Rel}_{i j}}$ are all set to $0.010,-0.010,0.015$ or -0.015 so that abovementioned restrictions on the means of the relationship effects are also satisfied.

Four different sample sizes are considered, $N=50, N=$ $150, N=300$, and $N=500$. In the Bayesian approach we specified non-informative priors on the means (i.e., family mean, the role-specific perceiver and target means, and the relationship means) that each followed a normal distribution with a mean of zero and a precision of 0.0001. For the precision matrices, we defined the following priors

$$
\begin{gathered}
\tau_{\mathrm{FE}} \sim \Gamma(0.01,0.01), \\
\Sigma_{\mathrm{PerTar}_{i}}^{-1} \sim W(2, \omega I),
\end{gathered}
$$

with the prior for the precision of the family effect gamma-distributed with a scale and shape parameter of 0.01 and the prior for the precision matrix of the perceiver and target effects for each role Wishart distributed. The two parameters of the Wishart prior are the degrees of freedom $(=2)$ and the scale matrix $(=\omega I)$. To allow for a sensitivity analysis on the scale matrix of the Wishart prior, we varied $\omega$ between $0.2,1$, and 5 . By considering those three different scale matrices, we can assess the impact on the (un)biasedness of the variance components of the SRM. We consider large perceiver variances and small target variances, because the influential character of the Wishart prior is especially apparent when the true variances are close to zero (Schuurman et al., 2016). We generate 1,000 simulated datasets for each condition. For the ML-estimator these data sets are analyzed using Mplus and for the Bayesian approach they are analyzed in R ( $\mathrm{R}$ Development Core Team, 2017) using rjags (Plummer, 2016). In the former, the estimates are obtained using Monte-Carlo integration with 5,000 integration points. In the Bayesian approach Gibbs sampling is used with an adaptation period of 1,000 iterations, 3 chains of 10,000 iterations and a thinning factor of 15 . Lastly, for the Bayesian approach the mean of the posterior distribution is used as an estimator of the SRM means and variances.

The performances of the two frameworks are compared in terms of bias, coverage and precision. First, the bias is assessed by taking the difference between the median of the estimated parameter across simulations and the underlying true value using a boxplot. Note that we use the median instead of the mean to minimize the impact of outliers. The empirical coverage is calculated as the proportion of times the true value lies in the $95 \%$ confidence interval of the estimated parameter. Further, the precision is calculated using the median absolute deviation (MAD) from the true value, which is a more robust measure than the mean squared error (MSE). Note that in the body of text we do not provide tables with results for every single SRM-effect, but rather opt to present only the results for the family effect, one perceiver and one target effect. This, because the underlying values for all the perceiver and target effects are quasi the same.

\section{Results}

\section{Bias}

Figures 3 and 4 present the absolute bias for the means and the variances for the family effect and the perceiver and target effect of the mother. Both the ML-estimator and the Bayesian estimator retrieve the SRM parameters of the mean-structure under all the conditions well, except for the Bayesian estimator with $\omega=5$ of the family mean. All the methods perform relatively well in the estimation of the variances of the family and perceiver effect. However, the Bayesian estimators tend to result in biased estimates for small target variances, especially when $\omega=$ 1 or $\omega=5$. This observation is not very surprising, since the influential character of the scale matrix of the Wishart is more apparent when the true variances are close to zero (Schuurman et al., 2016). These results clearly show that the Wishart can be a very influential and informative prior. However, since the bias disappears, albeit gradually, when $\omega=0.2$ with increasing sample size, we conject that the Wishart prior does result in asymptotically unbiased estimates for the variances. No evidence for bias is found for the ML-estimates of the variances under all sample sizes.

Note that other priors could have been used, that could potentially lead to more accurate estimates of the variance components. First, there are some non-informative priors that are based on the Wishart distribution: the scaled inverse (Gelman \& Hill, 2007; O’Malley \& Zaslavsky, 2005) and the hierarchical inverse Wishart (Huang \& Wand, 2013). In our setting, however, those did not result in stable convergent chains. Second, one could make use of separate non-informative priors for the variances and covariances by applying the separation method of Barnard, McCulloch, and Meng (2000). The separation technique is, however, not ideal when performing Gibbs sampling due to its assumption of conditional conjugacy (Alvarez, Niemi, \& Simpson, 2014). Given these computational restrictions, the Wishart prior is in our opinion the best option available in this Bayesian sampling scenario.

Also ML-estimation is not without its downsides, since Mplus failed to converge in $6.6-16.4 \%$ of the datasets. More 
(A)
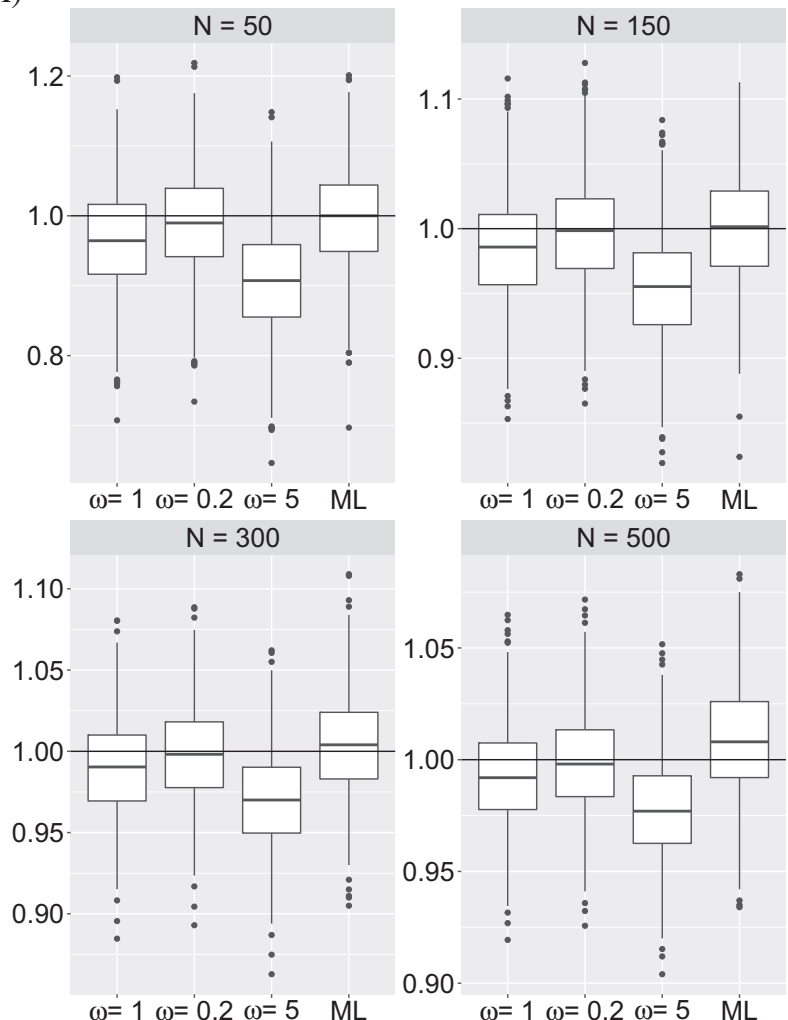

(C)

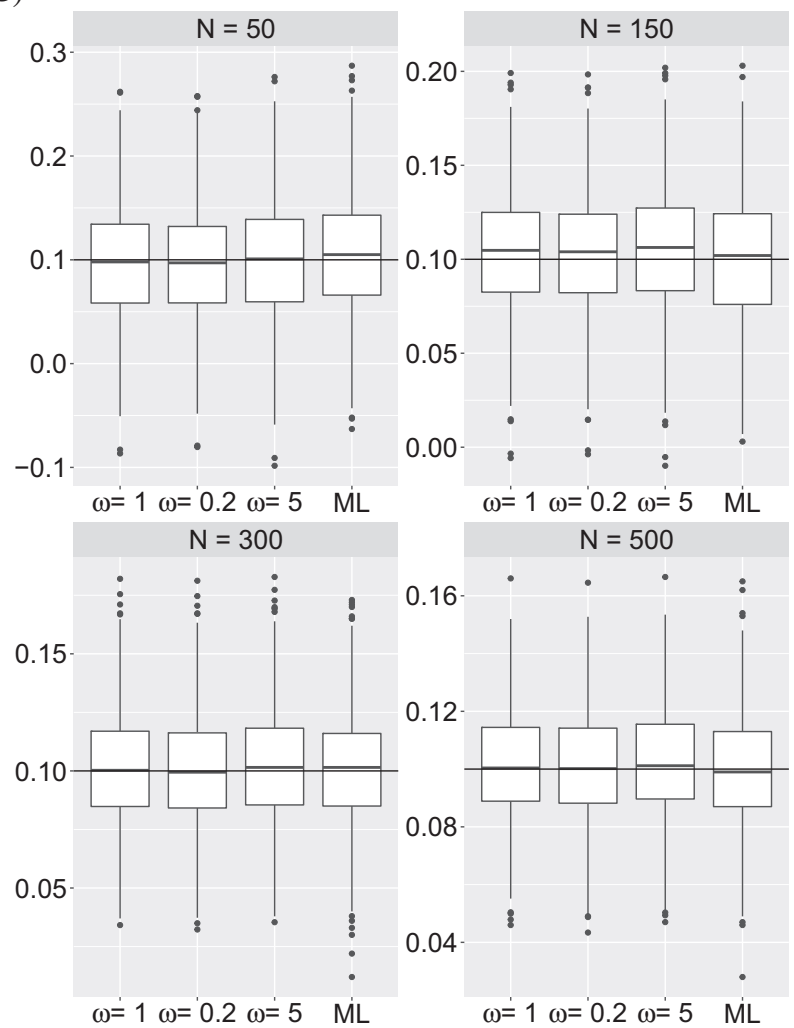

(B)

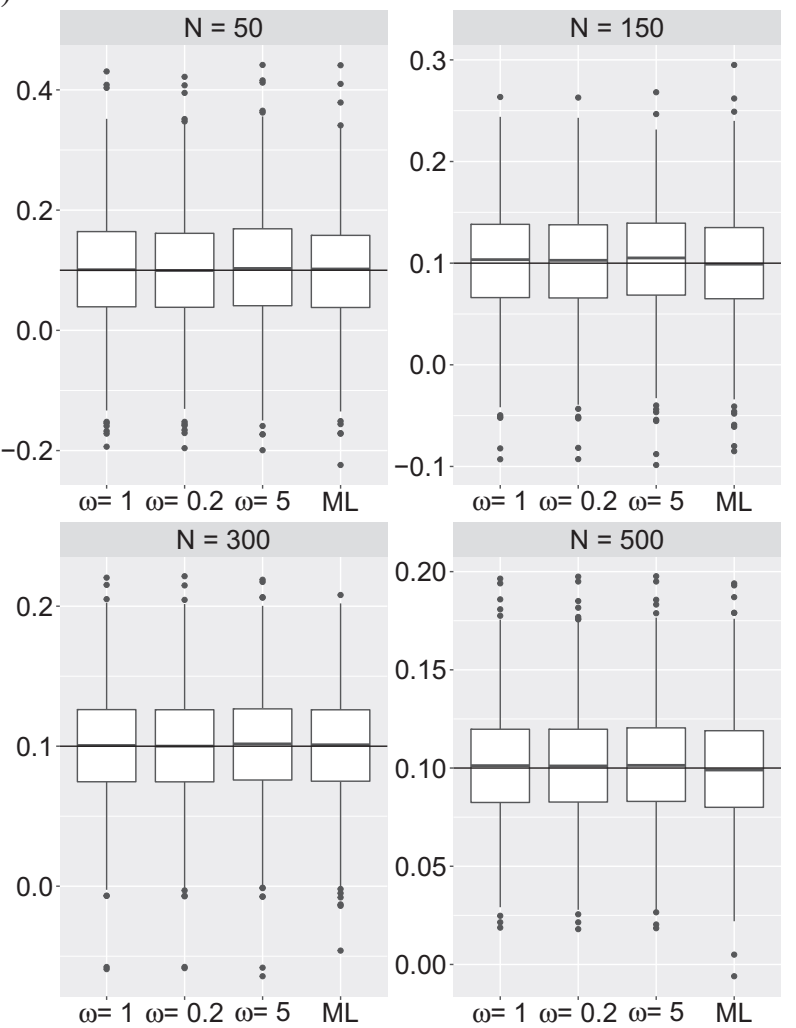

Figure 3. Simulation results for bias on the means of the SRM effects, where $\omega=1,0.2$, and 5 each refer to the different Bayesian approaches and $\mathrm{ML}$ refers to $\mathrm{ML}$ estimation (Black line = true value). (A) Family effect; (B) Perceiver effect of the mother; (C) Target effect of the mother. 
(A)

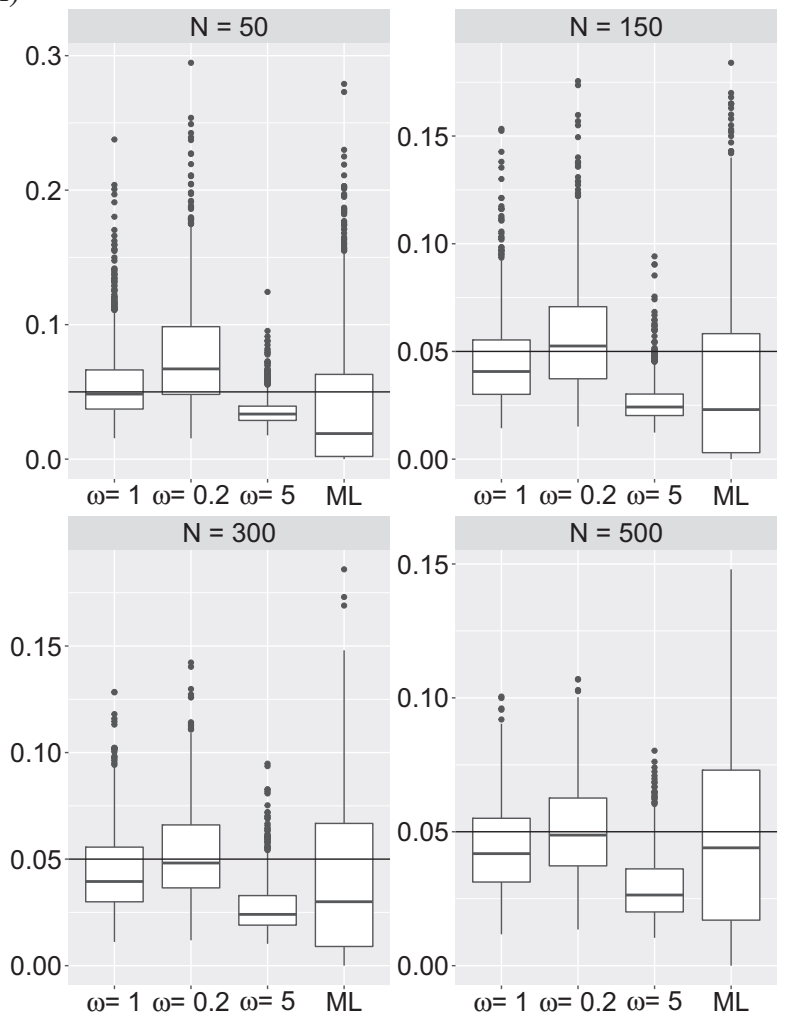

(B)

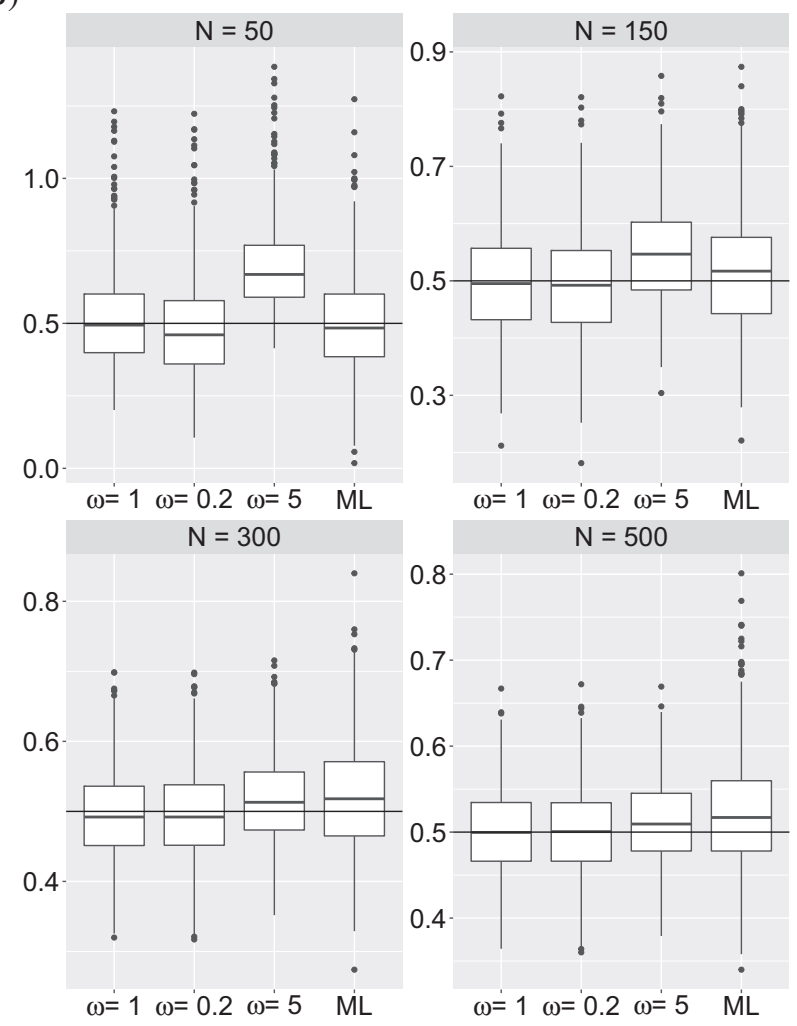

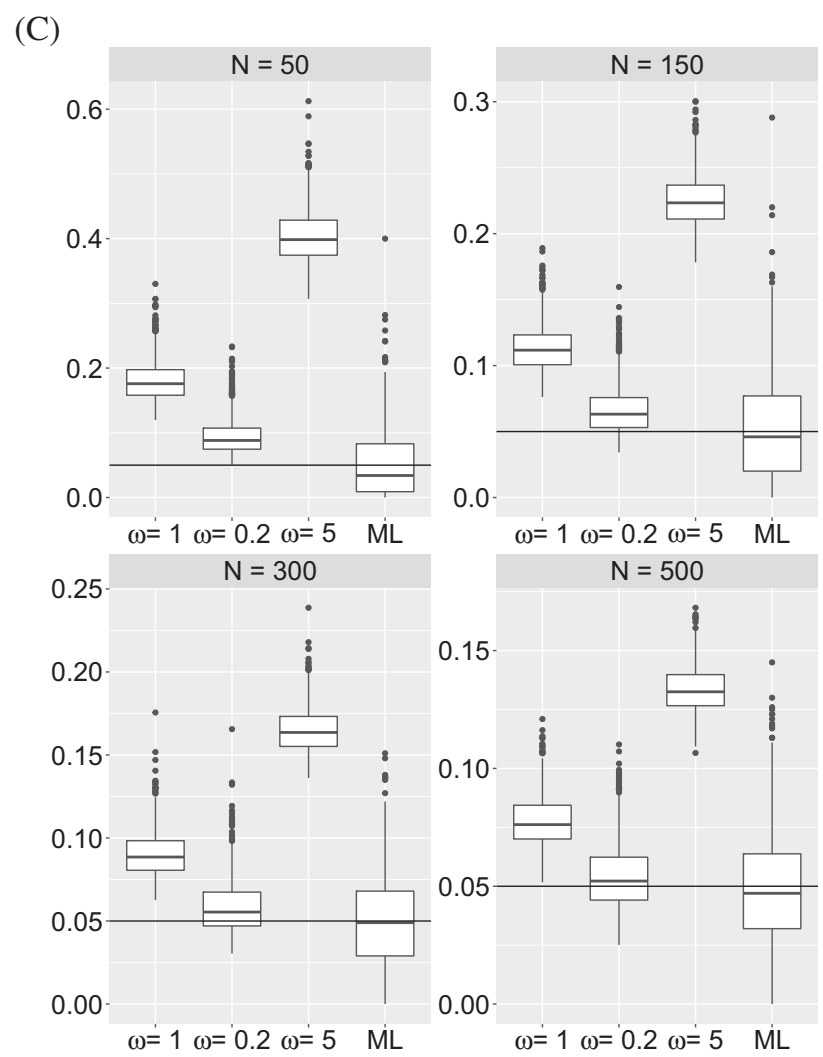

Figure 4. Simulation results for bias on the variances of the SRM effects, where $\omega=1,0.2$, and 5 each refer to the different Bayesian approaches and $M L$ refers to $M L$ estimation (Black line = true value). (A) Family effect; (B) Perceiver effect of the mother; (C) Target effect of the mother. 
convergency issues are encountered in smaller samples. These issues could be circumvented by increasing the number of integration points, however, that increases the computation time heavily.

\section{Coverage}

An overview of the coverage rates can be found in Table 1 . None of the conditions displays a good coverage probability in small samples. With increasing sample size all the conditions tend to approach the aspired probability coverage rates for the means, except for the Bayesian approach with $\omega=5$. Only the Bayesian approach with $\omega=0.2$ reaches the desired coverage probability rates for the variance parameters with increasing sample size, except for the coverage probability of the small target variance. The other conditions all show over- or under-coverage for each of the variance parameters.

\section{Precision}

The MAD is shown in Table 2. All the conditions have about the same precision for parameters of the mean-structure. As expected this precision improves as the sample size increases. For the variance parameters, the Bayesian approach with $\omega=0.2$ results in the most precise estimates. However, this discrepancy in precision for the variance parameters with the ML-estimator becomes smaller in larger samples.

\section{Conclusion}

We conclude that the ML-estimator tends to result in less biased estimators of the SRM parameters, while the Bayesian approach tends to result in more precise estimators. However, the performance of both the ML-estimator and the Bayesian estimator with $\omega=0.2$ is very comparable for large samples. The latter may thus be an acceptable alternative if the ML-approach fails to converge.

\section{Case Study}

\section{Results}

\section{Overall Analysis}

It is well-known that parent-adolescent interactions and the affective climate of the family have an impact on the adolescent's social development and personal functioning (Ge \& Conger, 1999; Kim, Conger, Lorenz, \& Elder, 2001; Paley, Conger, \& Harold, 2000). Not surprisingly, various studies have shown that family co-activity, which can be seen as an interaction with a positive affect, has such an effect (e.g., Hodge et al., 2017; Zabriskie \& McCormick, 2001). But what are the sources of the variability in coactivity between families? The SRM can be used to formulate an answer on this question.
Table 1. Simulation results for the coverage rate of the ML-estimator and Bayesian estimator for SRM-effects in blocked design

\begin{tabular}{|c|c|c|c|c|}
\hline Condition & $N=50$ & $N=150$ & $N=300$ & $N=500$ \\
\hline \multicolumn{5}{|c|}{ Family effect mean } \\
\hline$\omega=0.2$ & 0.965 & 0.956 & 0.951 & 0.938 \\
\hline$\omega=1$ & 0.937 & 0.936 & 0.930 & 0.934 \\
\hline$\omega=5$ & 0.844 & 0.829 & 0.821 & 0.838 \\
\hline $\mathrm{ML}$ & 0.933 & 0.945 & 0.950 & 0.912 \\
\hline \multicolumn{5}{|c|}{ Perceiver effect mean } \\
\hline$\omega=0.2$ & 0.944 & 0.950 & 0.949 & 0.951 \\
\hline$\omega=1$ & 0.950 & 0.950 & 0.950 & 0.953 \\
\hline$\omega=5$ & 0.967 & 0.953 & 0.954 & 0.953 \\
\hline $\mathrm{ML}$ & 0.954 & 0.951 & 0.948 & 0.943 \\
\hline \multicolumn{5}{|c|}{ Target effect mean } \\
\hline$\omega=0.2$ & 0.978 & 0.957 & 0.960 & 0.948 \\
\hline$\omega=1$ & 0.988 & 0.972 & 0.972 & 0.952 \\
\hline$\omega=5$ & 0.996 & 0.990 & 0.983 & 0.964 \\
\hline $\mathrm{ML}$ & 0.941 & 0.951 & 0.937 & 0.959 \\
\hline \multicolumn{5}{|c|}{ Family effect variance } \\
\hline$\omega=0.2$ & 0.991 & 0.966 & 0.941 & 0.932 \\
\hline$\omega=1$ & 0.998 & 0.959 & 0.919 & 0.898 \\
\hline$\omega=5$ & 0.999 & 0.736 & 0.594 & 0.598 \\
\hline $\mathrm{ML}$ & 0.612 & 0.621 & 0.667 & 0.677 \\
\hline \multicolumn{5}{|c|}{ Perceiver effect variance } \\
\hline$\omega=0.2$ & 0.903 & 0.934 & 0.952 & 0.954 \\
\hline$\omega=1$ & 0.957 & 0.946 & 0.951 & 0.956 \\
\hline$\omega=5$ & 0.995 & 0.990 & 0.973 & 0.970 \\
\hline $\mathrm{ML}$ & 0.870 & 0.917 & 0.908 & 0.875 \\
\hline \multicolumn{5}{|c|}{ Target effect variance } \\
\hline$\omega=0.2$ & 1.000 & 1.000 & 0.992 & 0.986 \\
\hline$\omega=1$ & 0.920 & 0.623 & 0.704 & 0.803 \\
\hline$\omega=5$ & 0.000 & 0.000 & 0.000 & 0.000 \\
\hline$M L$ & 0.731 & 0.849 & 0.894 & 0.901 \\
\hline
\end{tabular}

Note. $\mathrm{ML}=$ maximum likelihood; SRM = social relations model.

Using the NEAD co-activity data, we fitted an SRM in the statistical software Mplus using ML estimation. First we made the assumption that the dyadic measurements are normally distributed. To assess this assumption, we can contrast the model-based predictions with the observed dyadic measurements (Kruschke, 2014). Not all the predictive distributions mimic the observed count distributions well (Figure 5).

Instead of a normal distribution, we next assume a Poisson distribution. Then the ML-estimates are obtained by making use of Monte-Carlo integration with 5,000 integration points. Again, a predictive check can be performed. Figure 5 reveals that this model fits much better. Estimates of the SRM parameters of the model assuming a Poisson distribution can be found in Table 3, as well as their 95\% credibility intervals (CI). Alternatively, the estimates of the parameters can be obtained by using Bayesian sampling methods. For this, we made use of the R-package 
Table 2. Simulation results for the MAD of the ML-estimator and Bayesian estimator for SRM-effects in blocked design

\begin{tabular}{|c|c|c|c|c|}
\hline Condition & $N=50$ & $N=150$ & $N=300$ & $N=500$ \\
\hline \multicolumn{5}{|c|}{ Family effect mean } \\
\hline$\omega=0.2$ & 0.049 & 0.028 & 0.020 & 0.015 \\
\hline$\omega=1$ & 0.054 & 0.030 & 0.020 & 0.016 \\
\hline$\omega=5$ & 0.094 & 0.046 & 0.031 & 0.024 \\
\hline $\mathrm{ML}$ & 0.048 & 0.029 & 0.020 & 0.018 \\
\hline \multicolumn{5}{|c|}{ Perceiver effect mean } \\
\hline$\omega=0.2$ & 0.062 & 0.036 & 0.026 & 0.019 \\
\hline$\omega=1$ & 0.062 & 0.036 & 0.026 & 0.018 \\
\hline$\omega=5$ & 0.066 & 0.037 & 0.025 & 0.018 \\
\hline $\mathrm{ML}$ & 0.060 & 0.035 & 0.025 & 0.019 \\
\hline \multicolumn{5}{|c|}{ Target effect mean } \\
\hline$\omega=0.2$ & 0.038 & 0.021 & 0.016 & 0.013 \\
\hline$\omega=1$ & 0.037 & 0.021 & 0.016 & 0.013 \\
\hline$\omega=5$ & 0.040 & 0.023 & 0.017 & 0.013 \\
\hline $\mathrm{ML}$ & 0.039 & 0.024 & 0.016 & 0.013 \\
\hline \multicolumn{5}{|c|}{ Family effect variance } \\
\hline$\omega=0.2$ & 0.021 & 0.016 & 0.015 & 0.013 \\
\hline$\omega=1$ & 0.014 & 0.016 & 0.016 & 0.014 \\
\hline$\omega=5$ & 0.017 & 0.026 & 0.026 & 0.024 \\
\hline $\mathrm{ML}$ & 0.045 & 0.039 & 0.034 & 0.028 \\
\hline \multicolumn{5}{|c|}{ Perceiver effect variance } \\
\hline$\omega=0.2$ & 0.116 & 0.064 & 0.043 & 0.034 \\
\hline$\omega=1$ & 0.101 & 0.063 & 0.043 & 0.034 \\
\hline$\omega=5$ & 0.169 & 0.065 & 0.040 & 0.034 \\
\hline $\mathrm{ML}$ & 0.107 & 0.070 & 0.052 & 0.043 \\
\hline \multicolumn{5}{|c|}{ Target effect variance } \\
\hline$\omega=0.2$ & 0.038 & 0.013 & 0.010 & 0.009 \\
\hline$\omega=1$ & 0.126 & 0.062 & 0.039 & 0.038 \\
\hline$\omega=5$ & 0.348 & 0.173 & 0.114 & 0.037 \\
\hline$M L$ & 0.040 & 0.029 & 0.020 & 0.017 \\
\hline
\end{tabular}

Note. $\mathrm{MAD}=$ median absolute deviation; $\mathrm{ML}=$ maximum likelihood; $\mathrm{SRM}=$ social relations model.

rjags (Plummer, 2016). The same non-informative priors as in the simulation study are specified for the means and the precision matrices of all the effects. Again, the scale matrix of the Wishart, $\omega$, was allowed to vary between $0.2,1$, and 5 . We opted for 3 MCMC (Markov Chain Monte Carlo) chains, 1,000 adaptation iterations, an extra 10,000 iterations, and the thinning factor was set at 15 . The chains of the three models mixed very well and converged clearly to a stable point (based on traceplots, Gelman-Rubin plots, and autocorrelation plots; results not shown).

The expected a posterior (EAP) or the mean of the posterior distribution of the SRM parameters for the three values of $\omega$ can be found in Table 3. However, given the results of the simulation study we will limit our discussion to the Bayesian estimates where $\omega=0.2$. Note that the exponent of the SRM means is presented, this to facilitate the interpretation. In all the families, 2.852 activities are on average perceived to have occurred in the past month. Additionally, the mothers report on average the most activities within the families: relative to other family members they perceive on average 1.447 times more different activities with other family members (i.e., their children).

Next, we can interpret the SRM variances by determining the VPC. The relative variance decomposition of the two models is shown in Figure 6. Based on the relative variance decomposition in Figure 6B, we find that the perceiver effects explain more variance of the co-activity behavior than the target effects. These results are in line with previous family research on affectivity (Eichelsheim, Dekovic, Buist, \& Cook, 2009). Typically, the family effect is found to be a small source of variance. However, here we notice that it explains a great part of the dyadic measurements when the mother is perceiver. This can be explained by the fact that both the perceiver and target effect of the mother have a small variance. Thus, the amount of perceiver and target variance of the mother does not vary a lot across families. This stable and high perception of activities concurs with how the society typically bestows the mother with the role of keeping the family together (Weaver \& Coleman, 2010).

\section{Group Analysis}

It is known that engaging in leisure activities can foster the cohesiveness in families and is therefore of importance to stepfamilies (Pylyser, Buysse, \& Loeys, 2017). We can therefore wonder whether the family dynamics of co-activity are different between non-divorced families and stepfamilies. An SRM analysis that compares the sources of variability in co-activity between non-divorced families and stepfamilies might give some insight into which main drivers foster family cohesiveness in stepfamilies compared to those in non-divorced families. Estimates of the SRM parameters in both groups of the ML and Bayesian approach can be found in Table 4 .

When comparing the relative variance decomposition of both groups, the differences between the two groups become most visible (Figure 7). The variance of the perceiver effects tends to explain more of the variance in co-activity across stepfamilies, while the family effect tends to explain more of the variance in the non-divorced families. The target effects in both types of family do not account for a lot of variance in the dyadic measurements of co-activity. An exception is the target effect of the stepfather, which explains more of the variance in the stepfamilies. The latter effect is very crucial to stepfamilies, since it embodies the children's perception of co-activity with their stepfather. Specifically, children tend to deduce feelings of mattering from such shared child-stepparent activities, which are essential to the family functioning (Ganong, Coleman, Fine, \& Martin, 1999; Pylyser et al., 2017). 
(A)

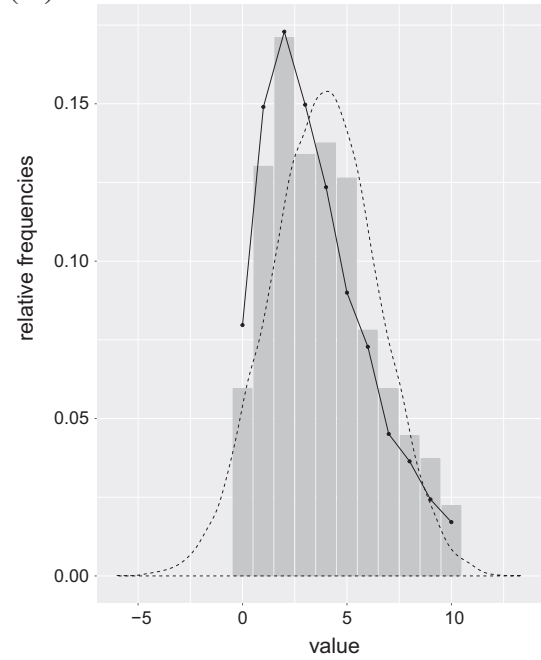

(D)

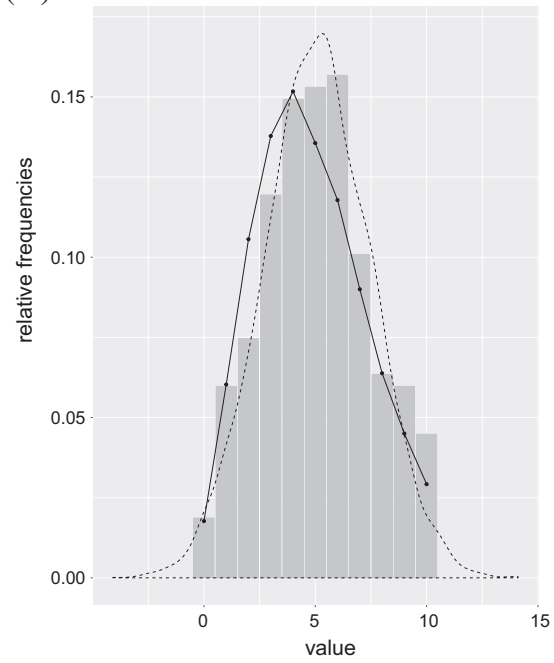

(B)

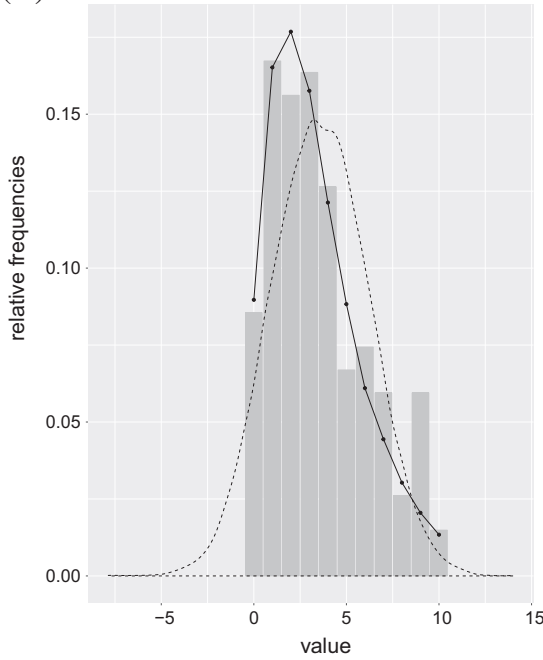

(E)

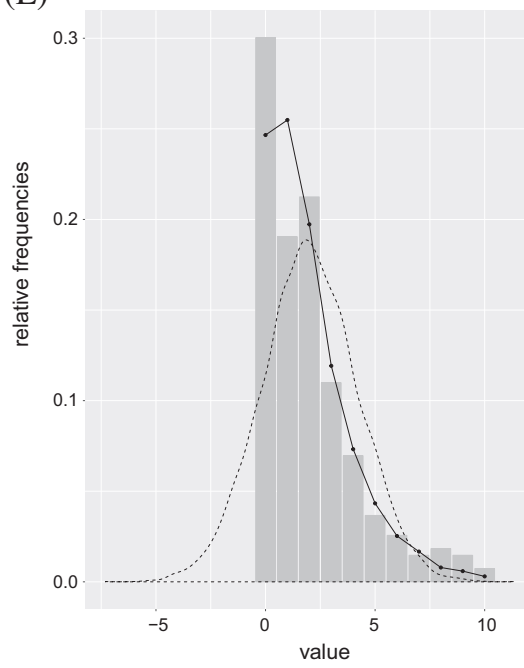

(C)

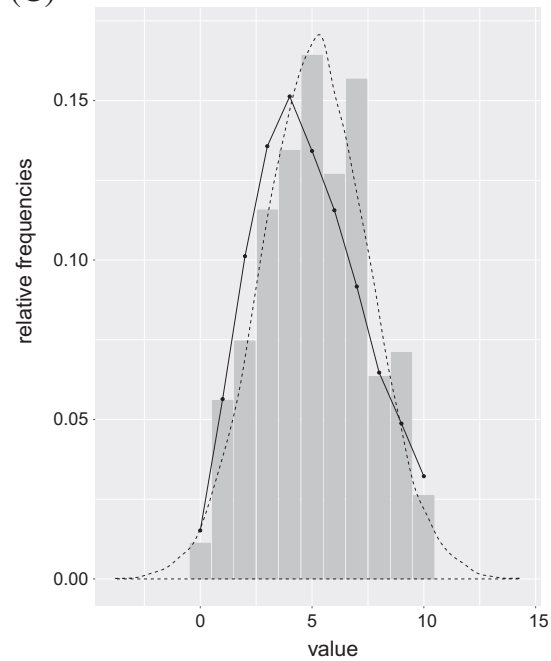

(F)

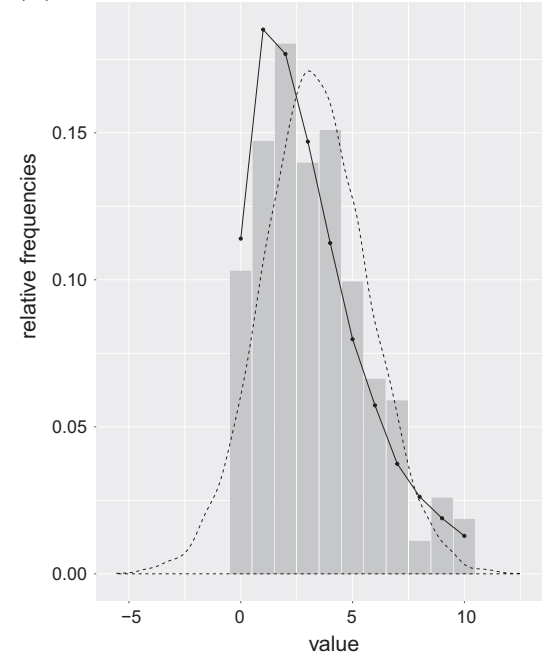

(G)

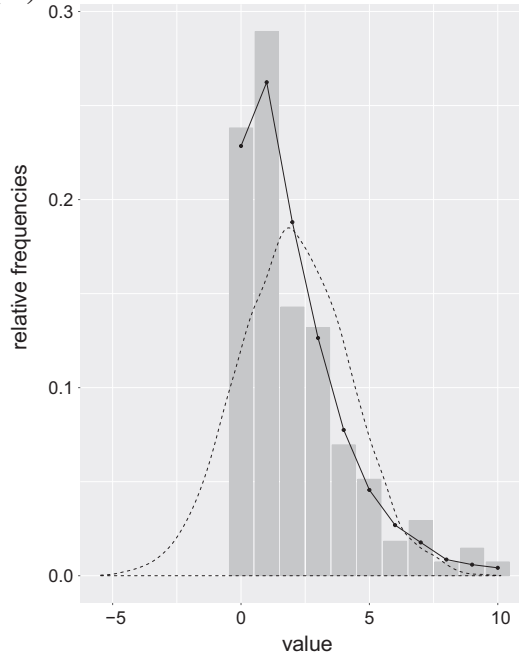

(H)

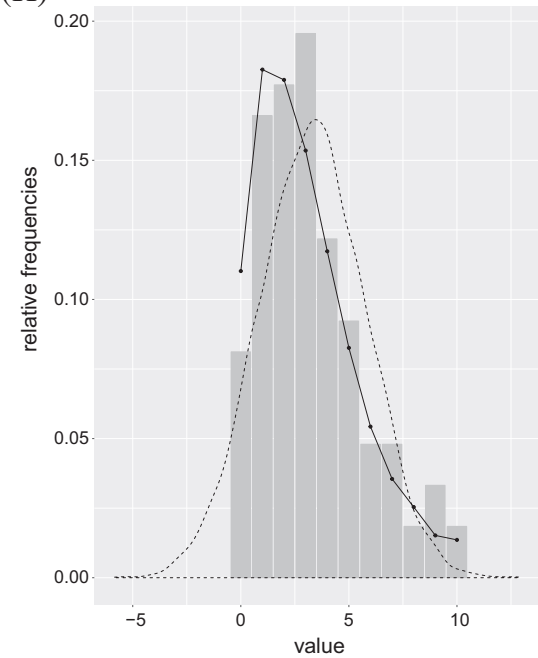

Figure 5. Observed data (bars) versus predictive check data (lines: dashed = continuous indicators; solid = count indicators). (A) F-C1 count; (B) F-C1 count; (C) M-C1 count; (D) M-C2 count; (E) C1-F count; (F) C1-M count; (G) C2-F count; (H) C2-M count. 
Table 3. Summary of general analysis of NEAD data. The exponent of the SRM means and the SRM (co-)variances are presented

\begin{tabular}{|c|c|c|c|c|c|c|c|c|}
\hline \multirow[b]{3}{*}{ Parameters } & \multicolumn{2}{|c|}{ ML-estimation } & \multicolumn{6}{|c|}{ Bayesian estimation } \\
\hline & \multicolumn{2}{|c|}{$\mathrm{ML}$} & \multicolumn{2}{|r|}{$\omega=0.2$} & \multicolumn{2}{|r|}{$\omega=1$} & \multicolumn{2}{|r|}{$\omega=5$} \\
\hline & Estimate & $\mathrm{Cl}$ & EAP & $\mathrm{Cl}$ & EAP & $\mathrm{Cl}$ & EAP & $\mathrm{Cl}$ \\
\hline \multicolumn{9}{|l|}{ SRM means } \\
\hline Family Effect & 2.863 & {$[2.686,3.053]$} & 2.852 & {$[2.692,3.013]$} & 2.821 & {$[2.666,2.982]$} & 2.747 & {$[2.596,2.907]$} \\
\hline Perceiver Effect M & 1.452 & {$[1.379,1.530]$} & 1.447 & {$[1.381,1.519]$} & 1.443 & {$[1.373,1.517]$} & 1.445 & {$[1.371,1.524]$} \\
\hline Perceiver Effect F & 0.938 & {$[0.885,0.994]$} & 0.940 & {$[0.890,0.992]$} & 0.944 & {$[0.893,0.997]$} & 0.947 & {$[0.894,1.003]$} \\
\hline Perceiver Effect $\mathrm{C} 1$ & 0.846 & {$[0.783,0.911]$} & 0.843 & {$[0.786,0.903]$} & 0.842 & {$[0.784,0.902]$} & 0.838 & {$[0.779,0.899]$} \\
\hline Perceiver Effect C2 & 0.869 & {$[0.806,0.938]$} & 0.872 & {$[0.811,0.934]$} & 0.872 & {$[0.811,0.935]$} & 0.871 & {$[0.810,0.937]$} \\
\hline Target Effect M & 1.114 & {$[1.064,1.166]$} & 1.119 & {$[1.068,1.174]$} & 1.128 & {$[1.072,1.186]$} & 1.139 & {$[1.075,1.203]$} \\
\hline Target Effect F & 0.659 & {$[0.623,0.697]$} & 0.657 & {$[0.622,0.692]$} & 0.651 & {$[0.615,0.688]$} & 0.641 & {$[0.602,0.682]$} \\
\hline Target Effect C1 & 1.196 & {$[1.168,1.225]$} & 1.195 & {$[1.153,1.239]$} & 1.197 & {$[1.153,1.244]$} & 1.203 & {$[1.150,1.257]$} \\
\hline Target Effect C2 & 1.139 & {$[1.107,1.171]$} & 1.137 & {$[1.096,1.183]$} & 1.137 & {$[1.091,1.185]$} & 1.138 & {$[1.085,1.195]$} \\
\hline \multicolumn{9}{|l|}{ SRM variances } \\
\hline Family Effect & 0.082 & {$[0.046,0.118]$} & 0.075 & {$[0.044,0.110]$} & 0.060 & {$[0.026,0.096]$} & 0.029 & {$[0.006,0.065]$} \\
\hline Perceiver Effect M & 0.028 & {$[-0.010,0.066]$} & 0.044 & {$[0.019,0.081]$} & 0.074 & {$[0.045,0.113]$} & 0.139 & {$[0.103,0.188]$} \\
\hline Perceiver Effect F & 0.262 & {$[0.178,0.346]$} & 0.259 & {$[0.177,0.353]$} & 0.271 & {$[0.189,0.367]$} & 0.325 & {$[0.237,0.433]$} \\
\hline Perceiver Effect C1 & 0.334 & {$[0.222,0.446]$} & 0.326 & {$[0.225,0.448]$} & 0.334 & {$[0.232,0.459]$} & 0.395 & {$[0.284,0.529)$} \\
\hline Perceiver Effect C2 & 0.320 & {$[0.216,0.424]$} & 0.311 & {$[0.212,0.426]$} & 0.310 & {$[0.208,0.426]$} & 0.352 & {$[0.251,0.476]$} \\
\hline Target Effect M & 0.000 & {$[0.000,0.000]$} & 0.024 & {$[0.012,0.042)$} & 0.054 & {$[0.034,0.083]$} & 0.128 & {$[0.092,0.174]$} \\
\hline Target Effect F & 0.069 & {$[-0.005,0.143]$} & 0.092 & {$[0.041,0.169]$} & 0.141 & {$[0.081,0.231]$} & 0.246 & {$[0.167,0.349)$} \\
\hline Target Effect C1 & 0.002 & {$[0.000,0.004]$} & 0.017 & {$[0.009,0.029)$} & 0.039 & {$[0.025,0.056]$} & 0.094 & {$[0.070,0.122)$} \\
\hline Target Effect C2 & 0.001 & {$[-0.001,0.003]$} & 0.017 & {$[0.009,0.028]$} & 0.039 & {$[0.026,0.057]$} & 0.098 & {$[0.073,0.131]$} \\
\hline \multicolumn{9}{|l|}{ SRM co-variances } \\
\hline Perceiver - Target M & -0.001 & {$[-0.009,0.007]$} & 0.002 & {$[-0.013,0.020]$} & 0.005 & {$[-0.014,0.027)$} & 0.014 & {$[-0.015,0.045]$} \\
\hline Perceiver - Target F & 0.094 & {$[0.028,0.16]$} & 0.089 & {$[0.032,0.156]$} & 0.088 & {$[0.031,0.158]$} & 0.098 & {$[0.034,0.174]$} \\
\hline Perceiver - Target C1 & 0.024 & {$[0.004,0.044]$} & 0.028 & {$[-0.005,0.065]$} & 0.033 & {$[-0.001,0.074]$} & 0.045 & {$[0.002,0.093)$} \\
\hline Perceiver - Target C2 & -0.013 & {$[-0.033,0.007)$} & -0.009 & {$[-0.042,0.025)$} & -0.003 & {$[-0.036,0.032]$} & 0.008 & {$[-0.031,0.050)$} \\
\hline
\end{tabular}

Note. $\mathrm{C} 1$ = child $1 ; \mathrm{C} 1$ = child $2 ; \mathrm{EAP}=$ expected a posterior; $\mathrm{F}=$ father; $\mathrm{M}=$ mother; $\mathrm{ML}=$ maximum likelihood; NEAD = Nonshared Environment in Adolescent Development; SRM = social relations model.

(A)

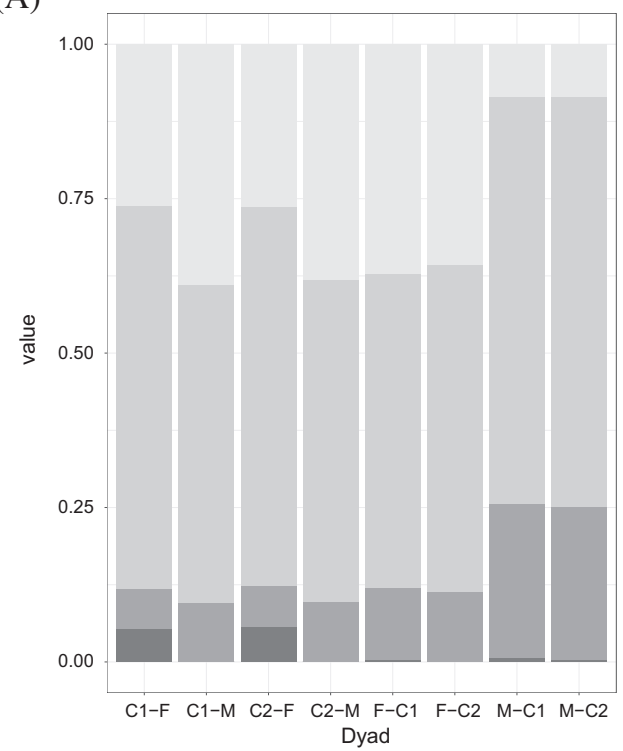

(B)

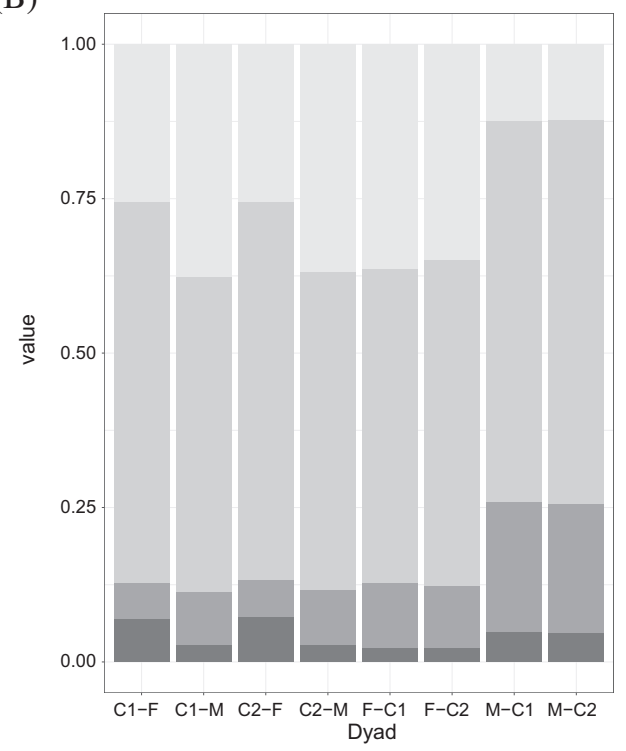

Figure 6. Relative variance decomposition (black = target, dark gray = family, gray = error, light gray = perceiver). (A) ML; $(B) \omega=0.2$. 
By calculating the ratio of the variances of each effect between both groups, the above described trends can be formally tested. The family effect varies significantly more in the ordinary families than in the stepfamilies (EAP of ratio $=3.742,95 \%$ CI $[1.255,10.661])$, illustrating that the ordinary families vary more in their average perception of co-activity within their intergenerational relationships. The perceiver effect of the older sibling (i.e., child 1) varies significantly more in the stepfamilies than the perceiver effect of the older sibling from ordinary families (Child 1: EAP of ratio $=0.416$, 95\% CI [0.141, 0.875]). This shows that those children from stepfamilies vary more in their perception of co-activity within their relationship to their mother and stepfather.

Next, we compare the means of the SRM effects between the two types of families. A significant ratio is present for the mean perceiver effects of the mother $($ Ratio $=0.828$, 95\% CI [0.753, 0.912]). Relative to the family mean, the mothers in ordinary families report 0.828 times the number of activities reported by the mothers from stepfamilies. Mothers from stepfamilies thus perceive more co-activity with their children relative to the family mean than the mothers from ordinary families. This is not surprising since the biological parent in stepfamilies, which is often the mother, undertakes the most action in organizing activities to create and sustain family ties (Pylyser et al., 2017). In contrast, the significant ratios of the father's perceiver and target effects suggest the opposite (Perceiver effect: Ratio $=1.119,95 \%$ CI $[1.007,1.243]$; Target effect: Ratio $=1.181,95 \%$ CI $[1.059,1.317])$. Relative to the family mean, the fathers from the ordinary families perceive 1.119 times more activities with their children than the stepfathers. Accordingly, the children from ordinary families perceive 1.181 times more activities with their father than children from stepfamilies. It seems that stepfathers put less effort into sharing activities with their stepchildren, which is especially true once they share a residence (Ganong et al., 1999). The large diversity in the tendency of stepfathers to build a relationship with their stepchildren, can furthermore explain why they perceive or elicit on average less co-activity with their stepchildren. This diversity in tendency can also explain why the perception of co-activity is so diverse in children from stepfamilies and, thus, why they have perceiver effects that explain larger amounts of variance. Lastly, there is on average more perceived co-activity in ordinary families than in stepfamilies (Ratio $=1.163,95 \%$ CI $[1.033,1.310])$.

\section{Discussion}

The SRM has received much attention in family literature. We presented a version of the family SRM that is appropriate for count data, hereby we extended the count SRM model proposed by Koster and Leckie (2014) to the family setting. Specifically, we proposed two approaches to estimate the SRM parameters for count data: the ML-estimator and the Bayesian estimator.

The simulation study showed that the ML-estimator actually results in less biased estimators for SRM-effects than the Bayesian estimator. This is especially true for small SRM variances. The presence of biased estimates of the variances by the Bayesian approach was not surprising given previous research on the Bayesian approach of the SRM (Lüdtke et al., 2012) and on the influential character of the scale matrix of the Wishart (Schuurman et al., 2016). Both approaches are, however, equally good in estimating the parameters of the mean-structure. The results also indicated that the Bayesian approach with a scale matrix that resembles the true underlying values performs just as good as the ML-estimator in large samples, a result which is in line with previous research on the definition of the scale matrix of the Wishart prior (Kass \& Natarajan, 2006). In that sense, a researcher could still opt to perform an SRM for count data using the Bayesian approach, since it still possesses some strengths compared to the frequentist approach. First, the ML-estimator requires more computational demand than the Bayesian approach and sometimes fails to converge. Second, the Bayesian approach can take into account prior information. And lastly, variance estimates cannot be negative. Furthermore, the applicability of these two approaches was illustrated using count dyadic measurements on perceived co-activity from the NEAD study. Note that we looked in two different modeling frameworks, the SEM and multilevel framework, because the two estimators are not yet directly available in both frameworks. Although we presented an SRM for count data from a block design, it should be noted that results also apply to count data from a round robin design.

There are some methodological limitations present in this paper. First, it might be interesting to study how other Bayesian sampling strategies, such as the No-U-Turn Sampler (NUTS; Hoffman \& Gelman, 2014), perform in estimating the SRM parameters. For example, in NUTS the separation strategy can be performed more easily. Second, a more extensive sensitivity analysis on the priors could be performed. Here, we limited our analysis to one hyperparameter of the Wishart distribution. Further research could study, for example, what the impact is of the other hyperparameter of the Wishart prior (i.e., the degrees of freedom) on the (co)variance estimates. Also, other adaptations of the Wishart prior, such as the uniform shrinkage prior (Chen \& Wehrly, 2016; Natarajan \& Kass, 2000), could be investigated. To that extent, researchers might also look into the impact of improper priors on the estimates of the variance components (Schuurman et al., 
Table 4. Summary of group analysis of NEAD data. The exponent of the SRM means and the SRM (co-)variances of ordinary families and stepfamilies are presented

\begin{tabular}{|c|c|c|c|c|c|c|c|c|}
\hline \multirow[b]{3}{*}{ Parameters } & \multicolumn{4}{|c|}{ Ordinary families } & \multicolumn{4}{|c|}{ Stepfamilies } \\
\hline & \multicolumn{2}{|r|}{$M L$} & \multicolumn{2}{|r|}{$\omega=0.2$} & \multicolumn{2}{|r|}{$M L$} & \multicolumn{2}{|r|}{$\omega=0.2$} \\
\hline & Estimate & $\mathrm{Cl}$ & EAP & $\mathrm{Cl}$ & EAP & $\mathrm{Cl}$ & EAP & $\mathrm{Cl}$ \\
\hline \multicolumn{9}{|l|}{ SRM means } \\
\hline Family Effect & 3.083 & {$[2.858,3.327]$} & 3.135 & {$[2.844,3.450]$} & 2.565 & {$[2.425,2.713]$} & 2.696 & {$[2.525,2.873]$} \\
\hline Perceiver Effect M & 1.305 & {$[1.231,1.383]$} & 1.269 & {$[1.180,1.368]$} & 1.571 & {$[1.495,1.652]$} & 1.532 & {$[1.442,1.630]$} \\
\hline Perceiver Effect $F$ & 1.003 & {$[0.937,1.074]$} & 1.020 & {$[0.936,1.104]$} & 0.905 & {$[0.854,0.959]$} & 0.911 & {$[0.849,0.976]$} \\
\hline Perceiver Effect C1 & 0.840 & {$[0.776,0.910]$} & 0.845 & {$[0.765,0.934]$} & 0.828 & {$[0.764,0.897]$} & 0.844 & {$[0.770,0.930]$} \\
\hline Perceiver Effect C2 & 0.909 & {$[0.838,0.987]$} & 0.915 & {$[0.826,1.008]$} & 0.849 & {$[0.783,0.919]$} & 0.849 & {$[0.775,0.926]$} \\
\hline Target Effect M & 1.044 & {$[0.991,1.100]$} & 1.059 & {$[0.980,1.139]$} & 1.176 & {$[1.116,1.239]$} & 1.158 & {$[1.085,1.231]$} \\
\hline Target Effect F & 0.732 & {$[0.691,0.776]$} & 0.730 & {$[0.676,0.790]$} & 0.598 & {$[0.560,0.637]$} & 0.618 & {$[0.575,0.663]$} \\
\hline Target Effect C1 & 1.155 & {$[1.114,1.197]$} & 1.152 & {$[1.086,1.224]$} & 1.230 & {$[1.196,1.265]$} & 1.224 & {$[1.167,1.282]$} \\
\hline Target Effect C2 & 1.134 & {$[1.090,1.181]$} & 1.124 & {$[1.054,1.197]$} & 1.157 & {$[1.123,1.192]$} & 1.141 & {$[1.088,1.20]$} \\
\hline \multicolumn{9}{|l|}{ SRM variances } \\
\hline Family Effect & 0.168 & {$[0.122,0.214]$} & 0.137 & {$[0.079,0.210]$} & 0.001 & {$[-0.001,0.003]$} & 0.046 & {$[0.014,0.085]$} \\
\hline Perceiver Effect M & 0.000 & {$[0.000,0.000]$} & 0.041 & {$[0.017,0.089]$} & 0.161 & {$[0.123,0.199]$} & 0.066 & {$[0.028,0.118]$} \\
\hline Perceiver Effect F & 0.233 & {$[0.153,0.313]$} & 0.162 & {$[0.065,0.295]$} & 0.457 & {$[0.365,0.549]$} & 0.286 & {$[0.181,0.422]$} \\
\hline Perceiver Effect $\mathrm{C} 1$ & 0.270 & {$[0.172,0.368]$} & 0.166 & {$[0.062,0.321]$} & 0.599 & {$[0.477,0.721]$} & 0.415 & {$[0.275,0.597]$} \\
\hline Perceiver Effect C2 & 0.296 & {$[0.192,0.400]$} & 0.175 & {$[0.064,0.336]$} & 0.496 & {$[0.396,0.596]$} & 0.357 & {$[0.231,0.513]$} \\
\hline Target Effect M & 0.000 & {$[0.000,0.000]$} & 0.040 & {$[0.016,0.086]$} & 0.004 & {$[-0.004,0.012]$} & 0.031 & {$[0.014,0.063]$} \\
\hline Target Effect $F$ & 0.004 & {$[-0.012,0.020]$} & 0.054 & {$[0.019,0.127]$} & 0.205 & {$[0.111,0.299]$} & 0.129 & {$[0.050,0.239]$} \\
\hline Target Effect C1 & 0.000 & {$[0.000,0.000]$} & 0.027 & {$[0.013,0.051]$} & 0.007 & {$[0.001,0.013]$} & 0.023 & {$[0.012,0.0414]$} \\
\hline Target Effect C2 & 0.003 & {$[-0.005,0.011]$} & 0.031 & {$[0.014,0.059]$} & 0.000 & {$[0.000,0.000]$} & 0.021 & {$[0.011,0.037]$} \\
\hline \multicolumn{9}{|l|}{ SRM co-variances } \\
\hline Perceiver - Target M & 0.000 & {$[0.000,0.000]$} & -0.001 & {$[-0.028,0.025]$} & 0.024 & {$[0.000,0.048]$} & 0.005 & {$[-0.020,0.034]$} \\
\hline Perceiver - Target F & 0.029 & {$[-0.041,0.099]$} & 0.010 & {$[-0.052,0.082]$} & 0.166 & {$[0.081,0.250]$} & 0.104 & {$[0.028,0.195]$} \\
\hline Perceiver - Target C1 & 0.002 & {$[-0.024,0.028]$} & 0.008 & {$[-0.032,0.060]$} & 0.063 & {$[0.031,0.095]$} & 0.043 & {$[-0.004,0.096]$} \\
\hline Perceiver - Target C2 & -0.030 & {$[-0.066,0.006]$} & -0.007 & {$[-0.055,0.041]$} & -0.004 & {$[-0.032,0.024]$} & -0.011 & {$[-0.056,0.036)$} \\
\hline
\end{tabular}

Note. $\mathrm{C} 1$ = child 1; $\mathrm{C1}$ = child 2; $\mathrm{EAP}=$ expected a posterior; $\mathrm{F}=$ father; $\mathrm{M}=$ mother; $\mathrm{ML}=$ maximum likelihood; NEAD = Nonshared Environment in Adolescent Development; SRM = social relations model.

(A)

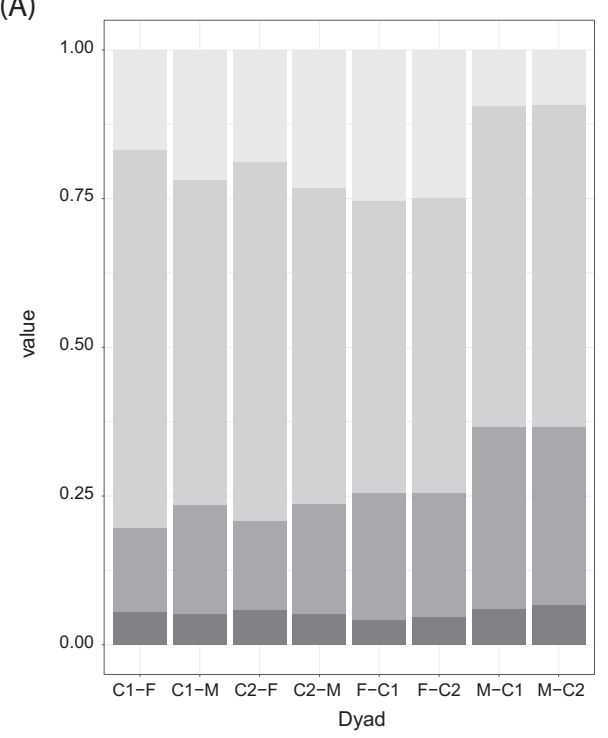

(B)

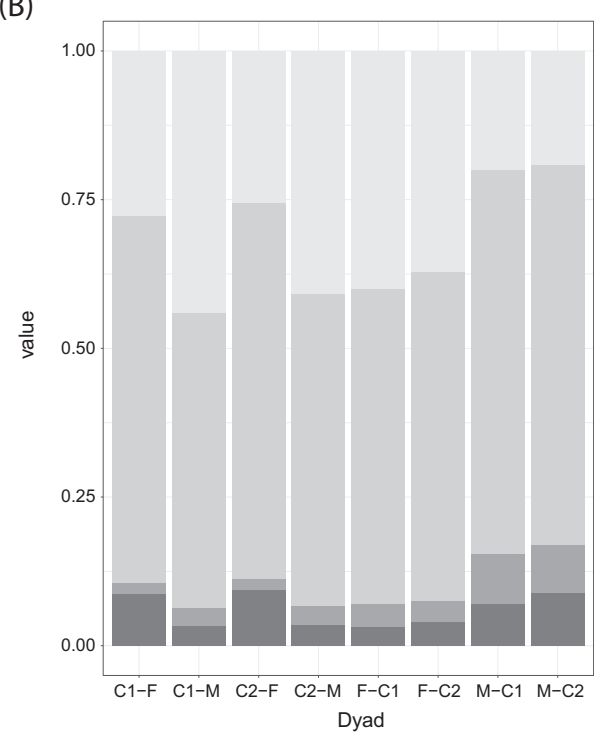

Figure 7. Relative variance decomposition of non-divorced and stepfamilies (black = target, dark gray = family, gray = error, light gray $=$ perceiver). (A) Non-divorced families $\omega=0.2$; (B) Stepfamilies $\omega=0.2$. 
2016). Further, it might be possible that in a real count data example overdispersion or zero-inflation is present. In that case it might be interesting to look into alternatives to the Poisson distribution such as the negative binomial distribution or zero-inflated count models. Lastly, it should be noted that we could also have opted to define and constrain the mean structure of the family SRM in another way. Here, we opted to constrain the means of the actor and target effects in an effect-coding manner relative to the family mean, which is the most conventional way to define the mean structure of the family SRM. However, other ways to define the mean structure, such as dummy-coding, are possible too. Perhaps another way of defining the constraints on the mean-structure might allow to model the family SRM more easily within the current multilevel software.

Despite these methodological limitations, we hope that the illustrative application and the methodological results of this paper will inspire family researchers to study dynamics in families on the basis of count dyadic measurements from a block or a round robin design.

\section{Electronic Supplementary Material}

The electronic supplementary material is available with the online version of the article at https://doi.org/ 10.1027/1614-2241/a000178

ESM 1. This file contains the MPlus and R code of the studies.

\section{References}

Alvarez, I., Niemi, J., \& Simpson, M. Bayesian inference for a covariance matrix. arXiv preprint arXiv:1408.4050

Austin, P. C., Stryhn, H., Leckie, G., \& Merlo, J. (2018). Measures of clustering and heterogeneity in multilevel Poisson regression analyses of rates/count data. Statistics in Medicine, 37, 572-589. https://doi.org/10.1002/sim.7532

Back, M. D., \& Kenny, D. A. (2010). The Social Relations Model: How to understand dyadic processes. Social and Personality Psychology Compass, 4, 855-870. https://doi.org/10.1111/ j.1751-9004.2010.00303.x

Barnard, J., McCulloch, R., \& Meng, X. L. (2000). Modeling covariance matrices in terms of standard deviations and correlations, with application to shrinkage. Statistica Sinica, 10, 1281-1311.

Bates, D. M., Mäechler, M., Bolker, B., \& Walker, S. (2015). Fitting linear mixed-effects models using Ime4. Journal of Statistical Software, 67, 1-48. https://doi.org/10.18637/jss.v067.i01

Chen, H. C., \& Wehrly, T. E. (2016). Approximate uniform shrinkage prior for a multivariate generalized linear mixed model. Journal of Multivariate Analysis, 145, 148-161. https://doi.org/10.1016/ j.jmva.2015.12.004

Cook, W. L. (1994). A structural equation model of dyadic relationships within the family system. Journal of Consulting and Clinical Psychology, 62, 500-509. https://doi.org/10.1037/ 0022-006X.62.3.500

Cook, W. L., \& Kenny, D. A. (2006). Examining the validity of selfreport assessments of family functioning: A question of the level of analysis. Journal of Family Psychology, 20, 209-216. https://doi.org/10.1037/0893-3200.20.2.209

Cox, M. J., \& Paley, B. (2003). Understanding families as systems. Current Directions in Psychological Science, 12, 193-196. https://doi.org/10.1111/1467-8721.01259

Eichelsheim, V., Dekovic, M., Buist, K., \& Cook, W. (2009). The Social Relations Model in family studies: A systematic review. Journal of Marriage and Family, 71, 1052-1069. https://doi.org/ 10.1111/j.1741-3737.2009.00652.x

Ganong, L., Coleman, M., Fine, M., \& Martin, P. (1999). Stepparents' affinity-seeking and affinity-maintaining strategies with stepchildren. Journal of Family Issues, 20, 299-327. https:// doi.org/10.1177/019251399020003001

Ge, X., \& Conger, R. D. (1999). Adjustment problems and emerging personality characteristics from early to late adolescence. American Journal of Community Psychology, 27, 429-459. https://doi.org/10.1023/A:1022238227937

Gelman, A., \& Hill, J. (2007). Data analysis using regression and multilevel/hierarchical regression. New York, NY: Cambride University Press.

Gill, P. S., \& Swartz, T. B. (2007). Bayesian analysis of dyadic data. American Journal of Mathematical and Management Sciences, 27, 73-92. https://doi.org/10.1080/01966324.2007. 10737689

Hetherington, E. M., \& Clingempeel, W. G. (1992). Coping with marital transitions: A family systems perspective. Monographs of the Society for Research in Child Development, 57, 1-14. https://doi.org/10.2307/1166050

Hetherington, E., Henderson, S. H., Reiss, D., Anderson, E. R. Bridges, M., Chan, R. W., ... Taylor, L. C. (1999). Adolescent siblings in stepfamilies: Family functioning and adolescent adjustment. Monographs of the Society for Research in Child Development, 64, 222-246. https://doi.org/10.1111/15405834.00045

Hodge, C. J., Duerden, M. D., Layland, E. K., Lacanienta, A., Goates, M. C., \& Niu, X. M. (2017). The association between family leisure and family quality of life: A meta-analysis of data from parents and adolescents. Journal of Family Theory \& Review, 9, 328-346. https://doi.org/10.1111/jftr.12202

Hoff, P. D. (2015). Dyadic data analysis with amen. Retrieved from https://arxiv.org/abs/1506.08237

Hoffman, M. D., \& Gelman, A. (2014). The No-U-Turn Sampler: Adaptively setting path lengths in Hamiltonian Monte Carlo. Journal of Machine Learning, 15, 1593-1623. Retrieved from https://arxiv.org/abs/1111.4246

Huang, A., \& Wand, M. P. (2013). Simple marginally noninformative prior distributions for covariance matrices. Bayesian Analysis, 8, 439-452.

Kashy, D. A., \& Kenny, D. A. (1990). Analysis of family research designs: A model of interdependence. Communication Research, 17, 462-482. https://doi.org/10.1177/009365090017004004

Kass, R. E., \& Natarajan, R. (2006). A default conjugate prior for variance components in generalized linear mixed models (comment on article by Browne and Draper). Bayesian Analysis, 1, 535-542. https://doi.org/10.1080/01621459.2000.10473916

Kelley, H. H. (1979). Personal relationships: Their structures and processes. Hillsdale, NJ: Erlbaum.

Kenny, D. A., Kashy, D. A., \& Cook, W. L. (2006). Dyadic data analysis. New York, NY: Guilford Press.

Kenny, D. A., \& La Voie, L. (1984). The Social Relations Model. Advances in Experimental Social Psychology, 18, 141-182. https://doi.org/10.1016/S0065-2601(08)60144-6 
Kim, K. J., Conger, R. D., Lorenz, F. O., \& Elder, G. H. (2001). Parent-adolescent reciprocity in negative affect and its relation to early adult social development. Developmental Psychology, 37, 775-790. https://doi.org/10.1037/0012-1649. 37.6.775

Koster, J. M., \& Leckie, G. (2014). Food sharing networks in lowland Nicaragua: An application of the social relations model to count data. Social Networks, 38, 100-110. https://doi.org/ 10.1016/j.socnet.2014.02.002

Koster, J. M., Leckie, G., Miller, A., \& Hames, R. (2015). Multilevel modeling analysis of dyadic network data with an application to Ye'kwana food sharing. American Journal of Physical Anthropology, 157, 507-512. https://doi.org/10.1002/ ajpa.22721

Kruschke, J. (2014). Doing Bayesian data analysis: A tutorial with $R$, JAGS, and Stan. New York, NY: Academic Press.

Lee, S. Y., \& Song, X. Y. (2004). Evaluation of the Bayesian and maximum likelihood approaches in analyzing structural equation models with small sample sizes. Multivariate Behavioral Research, 39, 653-686. https://doi.org/10.1207/ s15327906mbr3904_4

Loeys, T., Moerkerke, B., De Smet, O., \& Buysse, A. (2012). The analysis of zero-inflated count data: Beyond zero-inflated Poisson regression. British Journal of Mathematical and Statistical Psychology, 65, 163-180. https://doi.org/10.1111/ j.2044-8317.2011.02031.x

Lüdtke, O., Robitzsch, A., Kenny, D. A., \& Trautwein, U. (2012). A general and flexible approach to estimating the social relations model using Bayesian approaches. Psychological Methods, 18, 101-119. https://doi.org/10.1037/a0029252

Merkle, E. C., \& Rosseel, Y. (2018). blavaan: Bayesian structural equation models via parameter expansion. Journal of Statitstical Software, 85, 1-30. https://doi.org/10.18637/jss. v085.i04

Muthén, L. K., \& Muthén, B. O. (2012). Mplus user's guide: Statistical analysis with latent variables (7th ed.). Los Angeles, CA: Muthén \& Muthén.

Natarajan, R., \& Kass, R. E. (2000). Reference Bayesian approaches for generalized linear mixed models. Journal of the American Statistical Association, 95, 227-237. https://doi. org/10.1080/01621459.2000.10473916

Neiderhiser, J. M., Reiss, D., \& Hetherington, M. (2007). The Nonshared Environment in Adolescent Development Project: A longitudinal family study of twins and siblings from adolescence to young adulthood. Twin Research and Human Genetics, 10, 74-83. https://doi.org/10.1375/twin.10.1.74

Nevitt, J., \& Hancock, G. R. (2004). Evaluating small sample approaches for model test statistics in structural equation modeling. Multivariate Behavioral Research, 39, 439-478. https://doi.org/10.1207/S15327906MBR3903_3

O’Malley, A. J., \& Zaslavsky, A. M. (2005). Variance-covariance functions for domain means of ordinal survey items. Survey Methodology, 31, 169-182.

Paley, B., Conger, R. D., \& Harold, G. T. (2000). Parents' affect, adolescent cognitive representations, and adolescent social development. Journal of Marriage and Family, 62, 761-776. https://doi.org/10.1111/j.1741-3737.2000.00761.x

Pinheiro, J., Bates, D., DebRoy, S., \& Sarkar, D., R Core Team. (2018). nlme: Linear and nonlinear mixed effects models. (R package version 3.1-137). Retrieved from https://CRAN.R-project.org/ package $=n(m e$

Plummer, M. (2009). JAGS: Just another Gibbs sampler. Retrieved from https://sourceforge.net/projects/mcmc-jags

Plummer, M. (2016). rjags: Bayesian Graphical Models using MCMC. (R package version 4-6). Retrieved from https://CRAN. $\mathrm{R}$-project.org/package $=$ rjags
Pylyser, C., Buysse, A., \& Loeys, T. (2017). Stepfamilies doing family: A meta-ethnography. Family Process,. Advance online publication. https://doi.org/10.1111/famp.12293

Rasbash, J., Jenkins, J., O'Connor, T. G., Tackett, J., \& Reiss, D. (2011). A social relations model of observed family negativity and positivity using a genetically informative sample. Journal of Personality and Social Psychology, 100, 474-491. https://doi. org/10.1037/a0020931

R Development Core Team. (2017). R: A language and environment for statistical computing. Vienna, Austria: R Foundation for Statistical Computing.

Reis, H. T., Collins, W. A., \& Berscheid, E. (2000). The relationship context of human behavior and development. Psychological bulletin, 126, 844-872. https://doi.org/10.1037/0033-2909.126. 6.844

Rosseel, Y. (2012). lavaan: An R package for structural equation modeling. Journal of Statistical Software, 48, 1-36. https://doi. org/10.18637/jss.v048.i02

Rovine, M. J., \& Molenaar, P. C. (2000). A structural modeling approach to a multilevel random coefficients model. Multivariate Behavioral Research, 35, 51-88. https://doi.org/10.1207/ S15327906MBR3501_3

Schuurman, N. K., Grasman, R. P. P. P., \& Hamaker, E. L. (2016). A comparison of inverse-wishart prior specifications for covariance matrices in multilevel autoregressive models. Multivariate Behavioral Research, 51, 185-206. https://doi.org/10.1080/ 00273171.2015 .1065398

Snijders, T. A., \& Kenny, D. A. (1999). The social relations model for family data: A multilevel approach. Personal Relationships, 6, 471-486. https://doi.org/10.1111/j.1475-6811.1999.tb00204.x

Stas, L., Schönbrodt, F., \& Loeys, T. (2015). Getting the most out of family data with the R-package SRM. Journal of Family Psychology, 29, 263-275. https://doi.org/10.1037/fam0000058

Tuerlinckx, F., Rijmen, F., Verbeke, G., \& De Boeck, P. (2006). Statistical inference in generalized linear mixed models: A review. British Journal of Mathematical and Statistical Psychology, 59, 225-255. https://doi.org/10.1348/000711005X79857

Weaver, S. E., \& Coleman, M. (2010). Caught in the middle: Mothers in stepfamilies. Journal of Social and Personal Relationships, 27, 305-326. https://doi.org/10.1177/0265407510361729

Zabriskie, R. B., \& McCormick, B. P. (2001). The influences of family leisure patterns on perceptions of family functioning. Family Relations, 50, 281-289. https://doi.org/10.1111/j.17413729.2001.00281.x

\section{History}

Received October 24, 2018

Revision received June 13, 2019

Accepted July 12, 2019

Published online December 16, 2019

\section{Acknowledgments}

In the present study, data of the NEAD (Nonshared Environment in Adolescent Development) study were used.

\section{Funding}

This work has been supported by Fonds Wetenschappelijk Onderzoek (G020115N) to Tom Loeys.

\section{Justine Loncke}

Faculty of Psychology and Educational Sciences

University of Ghent

Henri Dunantlaan 1

9000 Ghent

Belgium

justine.loncke@ugent.be 
Justine Loncke is doctoral researcher at the Department of Data Analysis, Faculty of Psychology and Educational Sciences, Ghent University, Belgium. Her primary research interests include dyadic data analysis, structural equation modeling and multilevel modeling.

William Cook is formally Associate Director, Center for Psychiatric Research, Maine Medical Center, Portland, Maine. His current interest is providing statistical support to graduate students using dyadic data analysis in their theses.
Jenae M. Neiderhiser is a Distinguished Professor of Psychology and Human Development and Family Studies at Penn State University, USA. Her primary research interests are on the interplay of genes and environments in the context of the family and development across the lifespan.

Tom Loeys is professor at the Department of Data Analysis, Faculty of Psychology and Educational Sciences, Ghent University, Belgium. His primary research interests include dyadic data analysis, causal mediation analysis and multilevel modeling. 